\title{
BOUNDED KÄHLER CLASS RIGIDITY OF ACTIONS ON HERMITIAN SYMMETRIC SPACES
}

\author{
By MARC BURGER AND AlESSANDRA IOZZI
}

Dedicated to the memory of Bob Brooks

\begin{abstract}
We prove that the conjugacy class of a Zariski dense representation $\pi: \Gamma \rightarrow \mathrm{PU}(p, q)$, $q>p \geqslant 1$, of a finitely generated group $\Gamma$ is completely determined by the pull-back via $\pi$ of a bounded cohomology class in $\mathrm{H}_{\mathrm{cb}}^{2}(\mathrm{PU}(p, q), \mathbb{R})$ defined in terms of the Kähler form on the associated symmetric space. Under the assumption that $\mathrm{H}_{\mathrm{b}}^{2}(\Gamma, \mathbb{R})$ is finite dimensional, we show that, up to equivalence, there is only a finite number of such representations for fixed $q>p \geqslant 1$; moreover, under the hypothesis that $\mathrm{H}_{\mathrm{b}}^{2}(\Gamma, \mathbb{R})$ injects into $\mathrm{H}^{2}(\Gamma, \mathbb{R})$, we estimate the total number of such representations (for all $q>p \geqslant 1$ ) to be bounded above by $\operatorname{dim}_{\mathbb{R}} \mathrm{H}^{2}(\Gamma, \mathbb{R})$.
\end{abstract}

(C) 2004 Elsevier SAS

RÉSUMÉ. - On montre que la classe de conjugaison d'une représentation d'image Zariski dense $\pi: \Gamma \rightarrow \mathrm{PU}(p, q), q>p \geqslant 1$ d'un groupe de type fini $\Gamma$ est déterminée par l'image inverse par $\pi$ d'une classe de cohomologie bornée dans $\mathrm{H}_{\mathrm{cb}}^{2}(\mathrm{PU}(p, q), \mathbb{R})$ déduite de la forme de Kähler de l'espace symétrique hermitien associé. Lorsque $\mathrm{H}_{\mathrm{b}}^{2}(\Gamma, \mathbb{R})$ est de dimension finie, on démontre qu'il n'y a, à équivalence près, qu'un nombre fini de représentations d'image Zariski dense pour $q>p \geqslant 1$ fixés; de plus, si l'on suppose que l'application naturelle $\mathrm{H}_{\mathrm{b}}^{2}(\Gamma, \mathbb{R}) \rightarrow \mathrm{H}^{2}(\Gamma, \mathbb{R})$ est injective, alors le nombre total de telles représentations (pour tous $q>p \geqslant 1$ ) est borné par $\operatorname{dim}_{\mathbb{R}} \mathrm{H}^{2}(\Gamma, \mathbb{R})$.

(c) 2004 Elsevier SAS

\section{Introduction}

The purpose of this paper is to introduce and study bounded analogues of classical invariants attached to isometric group actions on Hermitian symmetric spaces.

Let $\mathcal{X}$ be a Hermitian symmetric space (of non-compact type), that is a symmetric space admitting a complex structure invariant under the connected component $G$ of the group of isometries Iso $(\mathcal{X})$ of $\mathcal{X}$, and let $\Gamma$ be a group. Then the second continuous cohomology group $\mathrm{H}_{\mathrm{c}}^{2}(G, \mathbb{R})$ of $G$ with real coefficients is a vector space of dimension the number of irreducible factors of $\mathcal{X}$ [19] and, fixing a continuous class $\kappa$ on $G$, one obtains for every homomorphism $\pi: \Gamma \rightarrow G$ an invariant $\pi^{*}(\kappa) \in \mathrm{H}_{\mathrm{c}}^{2}(\Gamma, \mathbb{R})$, well defined and constant on the (topological) connected components of the representation variety $\operatorname{Hom}(\Gamma, G)$. For example, if $\Gamma=\pi_{1}(S)$ is the fundamental group of a compact oriented surface of genus at least 2, the Toledo invariant, which is the evaluation of $\pi^{*}(\kappa)$ on the fundamental class of $S$, does, in certain cases, distinguish the connected components of $\operatorname{Hom}(\Gamma, G)$, and, when maximal, contains substantial information 
about $\pi$ (see [17,21,32-34,4,7]; see also [22, Section 1.1] for analogous results when $\Gamma$ is a lattice in $\mathrm{SU}(1, n))$.

Assuming now that $\mathcal{X}$ is irreducible, an explicit differentiable cocycle $c_{G}$ providing a generator $\kappa_{G} \in \mathrm{H}_{\mathrm{c}}^{2}(G, \mathbb{R})$ is given by

$$
c_{G}\left(g_{1}, g_{2}, g_{3}\right):=\int_{\Delta\left(g_{1} x_{0}, g_{2} x_{0}, g_{3} x_{0}\right)} \omega,
$$

where $\omega$ is the Kähler form on $\mathcal{X}$ and $\Delta(x, y, z)$ denotes an oriented smooth triangle in $\mathcal{X}$ with geodesic sides. The starting point of our investigation is the fact that $c_{G}$ is a bounded function, namely

$$
\left\|c_{G}\right\|_{\infty}=\pi r_{G}, \quad r_{G}=\operatorname{rank}_{\mathbb{R}}(G),
$$

(see [11] for classical groups and [10] for the general case). Thus, in the terminology of [9] and [27], $c_{G}$ defines a continuous bounded cohomology class $\kappa_{G}^{\mathrm{b}} \in \mathrm{H}_{\mathrm{cb}}^{2}(G, \mathbb{R})$, which corresponds to $\kappa_{G}$ under the canonical isomorphism $\mathrm{H}_{\mathrm{cb}}^{2}(G, \mathbb{R}) \stackrel{\simeq}{\rightarrow} \mathrm{H}_{\mathrm{c}}^{2}(G, \mathbb{R})$ (see [9]).

Hence, for every homomorphism $\pi: \Gamma \rightarrow G$ we obtain an invariant $\pi^{*}\left(\kappa_{G}^{\mathrm{b}}\right)$ called the bounded Kähler class of $\pi$, which lies in the second bounded cohomology group $\mathrm{H}_{\mathrm{b}}^{2}(\Gamma, \mathbb{R})$ of $\Gamma$. Echoing the work of É. Ghys on the bounded Euler class [14,15], and the treatment in [22] of Matsumoto's rigidity theorem [25], it is natural to ask which additional information the bounded Kähler class contains. In this direction we have the following:

THEOREM 1.1. - Let $G=\mathrm{PU}(p, q), \Gamma$ a finitely generated group, $\pi: \Gamma \rightarrow G$ a homomorphism, and $\pi^{*}\left(\kappa_{G}^{\mathrm{b}}\right) \in \mathrm{H}_{\mathrm{b}}^{2}(\Gamma, \mathbb{R})$ its bounded Kähler class. Assume that $1 \leqslant p<q$ and that $\pi$ has Zariski dense image. Then

(i) $\pi^{*}\left(\kappa_{G}^{\mathrm{b}}\right) \neq 0$; and

(ii) $\pi^{*}\left(\kappa_{G}^{\mathrm{b}}\right)$ determines $\pi$ up to $G$-conjugation.

Remark 1.2. - In Theorem 1.1, the condition that $p$ be different from $q$ is necessary, since if $\Gamma=\pi_{1}(S)$ is the fundamental group of a compact oriented surface $S$ of genus at least two and $\pi_{1}, \pi_{2}: \Gamma \rightarrow \operatorname{PSU}(p, p):=G$ are any two representations with maximal Toledo invariant, then $\pi_{1}^{*}\left(\kappa_{G}^{\mathrm{b}}\right)=\pi_{2}^{*}\left(\kappa_{G}^{\mathrm{b}}\right)$ (see the proof of [7, Proposition 2.1]).

Thus, for $G=\mathrm{PU}(p, q)$ with $p<q$, Theorem 1.1 gives us an injective map

$$
\begin{aligned}
K: \mathcal{R}_{\mathrm{Zd}}(\Gamma, G) & \rightarrow \mathrm{H}_{\mathrm{b}}^{2}(\Gamma, \mathbb{R}) \\
\pi & \mapsto \pi^{*}\left(\kappa_{G}^{\mathrm{b}}\right)
\end{aligned}
$$

defined on the set of $G$-conjugacy classes of representations of $\Gamma$ into $G$ with Zariski dense image, which is equivariant with respect to the canonical actions of $\operatorname{Aut}(\Gamma)$ on source and target. Recall that $\mathcal{R}_{\mathrm{Zd}}(\Gamma, G)$ is in a natural way the set of real points of an affine variety ([1, Proposition 8.2] and [31, Proposition 4.3 and Remark 4.4]).

In fact, Theorem 1.1 is a special case of a more general result to which we now turn. For ease of statement let us introduce the following terminology: we say that a representation $\pi: \Gamma \rightarrow G$ is of type $(p, q)$ if $G$ is isomorphic to $\mathrm{PU}(p, q)$. Moreover we say that $\pi_{1}: \Gamma \rightarrow G_{1}$ and $\pi_{2}: \Gamma \rightarrow G_{2}$ are equivalent if there is an isometry $T: \mathcal{X}_{1} \rightarrow \mathcal{X}_{2}$ between the corresponding symmetric spaces such that $\pi_{2}(\gamma)=T \pi_{1}(\gamma) T^{-1}$. Finally, the Hermitian symmetric spaces under consideration will always be equipped with their normalized Bergman metric, when considered as bounded symmetric domains. Then we have: 
THEOREM 1.3. - Let $\Gamma$ be a finitely generated group and let $\pi_{i}: \Gamma \rightarrow G_{i}$ be pairwise inequivalent representations of type $\left(p_{i}, q_{i}\right)$, for $1 \leqslant i \leqslant n$. Assume that $1 \leqslant p_{i}<q_{i}$ and that $\pi_{i}(\Gamma)$ is Zariski dense. Then the set

$$
\left\{\pi_{i}^{*}\left(\kappa_{G_{i}}^{\mathrm{b}}\right): 1 \leqslant i \leqslant n\right\} \subset \mathrm{H}_{\mathrm{b}}^{2}(\Gamma, \mathbb{R})
$$

is linearly independent over $\mathbb{Z}$.

Using Theorem 1.3 we show:

Corollary 1.4. - Let $\Gamma$ be a finitely generated group. Assume that $\mathrm{H}_{\mathrm{b}}^{2}(\Gamma, \mathbb{R})$ is finite dimensional, and fix $1 \leqslant p<q$. Then there are, up to equivalence, only finitely many representations $\Gamma \rightarrow \mathrm{PU}(p, q)$ with Zariski dense image.

Remark 1.5. - In fact, we prove a stronger result from which Corollary 1.4 follows, namely that the image in $\mathrm{H}_{\mathrm{b}}^{2}(\Gamma, \mathbb{R})$ under the map $K$ in (1.2) of any continuous injective path

$$
c: I \rightarrow \mathcal{R}_{\mathrm{Zd}}(\Gamma, G)
$$

from a non-empty open interval $I \subset \mathbb{R}$, contains an uncountable subset which is independent over $\mathbb{R}$ (Proposition 9.1).

Recall now that if $\mathrm{QH}(\Gamma)$ is the vector space of quasihomomorphisms of $\Gamma$, that is functions $f: \Gamma \rightarrow \mathbb{R}$ such that

$$
\sup _{a, b \in \Gamma}|f(a b)-f(a)-f(b)|<\infty
$$

then the kernel of the comparison map $\mathrm{H}_{\mathrm{b}}^{2}(\Gamma, \mathbb{R}) \rightarrow \mathrm{H}^{2}(\Gamma, \mathbb{R})$ is described by the quotient

$$
\mathrm{EH}_{\mathrm{b}}^{2}(\Gamma)=\mathrm{QH}(\Gamma) /\left(\ell^{\infty}(\Gamma) \oplus \operatorname{Hom}(\Gamma, \mathbb{R})\right),
$$

where we identify two quasihomomorphisms when they differ by a homomorphism plus a bounded function. Applying Theorem 1.1(i) to $\Gamma=\mathbb{F}_{2}$, the free group on two generators, and taking into account that $\mathrm{H}^{2}\left(\mathbb{F}_{2}, \mathbb{R}\right)=0$, we deduce that any homomorphism $\pi: \mathbb{F}_{2} \rightarrow \mathrm{PU}(p, q)$, $p<q$, with Zariski dense image gives rise in a geometric way to a quasihomomorphism $f_{\pi}: \mathbb{F}_{2} \rightarrow \mathbb{R}$, which is not at bounded distance from a homomorphism. Moreover, in view of Remark 1.5, Corollary 1.4 gives another proof of the fact that the second bounded cohomology group of a free group in at least two generators is infinite dimensional by providing a new geometric construction of an uncountable number of linearly independent bounded classes and hence of linearly independent (equivalence classes) of quasihomomorphisms in $\mathrm{EH}_{\mathrm{b}}^{2}(\Gamma)$ (see [5], and also [18] and [26], for the original construction): for example, Benoist's construction of $\varepsilon$ Schottky groups [2] shows that the space of discrete, faithful, Zariski dense realizations of $\mathbb{F}_{2}$ in $\mathrm{PU}(p, q)$ has, modulo conjugation, positive dimension; see also [20] for an example of an explicit continuous deformation into $\operatorname{PSU}(1,2)$ of $\mathbb{F}_{2}$ realized as the ideal triangle group in $\operatorname{PSU}(1,1)$.

Imposing a stronger hypothesis, we conclude from Theorem 1.3:

COROLLARY 1.6. - Assume that $\Gamma$ is finitely generated and that the map

$$
\mathrm{H}_{\mathrm{b}}^{2}(\Gamma, \mathbb{R}) \rightarrow \mathrm{H}^{2}(\Gamma, \mathbb{R})
$$

is injective. Then the number of inequivalent, Zariski dense representations of type $(p, q)$, $1 \leqslant p<q$, is bounded by $\operatorname{dim}_{\mathbb{R}} \mathrm{H}^{2}(\Gamma, \mathbb{R})$. 
The hypotheses that $\mathrm{H}_{\mathrm{b}}^{2}(\Gamma, \mathbb{R})$ is finite dimensional and that $\mathrm{H}_{\mathrm{b}}^{2}(\Gamma, \mathbb{R})$ injects into $\mathrm{H}^{2}(\Gamma, \mathbb{R})$ are thus intrinsic conditions on the bounded cohomology of $\Gamma$ guaranteeing strong rigidity properties. In the same vein, $\mathrm{N}$. Monod and Y. Shalom have shown that the condition $\mathrm{H}_{\mathrm{b}}^{2}\left(\Lambda, \ell^{2}(\Lambda)\right) \neq 0$ imposes on a group $\Lambda$ a rather strong rigid behavior in the context of orbit equivalence [28].

As sources of examples concerning Corollaries 1.4 and 1.6, we mention the following:

Examples 1.7. - (1) If $\Gamma<G_{1} \times G_{2}$ is a lattice in a product of compactly generated locally compact groups with dense projection onto each factor, one has the isomorphism [9]

$$
\mathrm{H}_{\mathrm{b}}^{2}(\Gamma) \simeq \mathrm{H}_{\mathrm{cb}}^{2}\left(G_{1}\right) \oplus \mathrm{H}_{\mathrm{cb}}^{2}\left(G_{2}\right) .
$$

In the particular case in which if $\Gamma<\operatorname{Aut}\left(\mathcal{T}_{1}\right) \times \operatorname{Aut}\left(\mathcal{T}_{2}\right)$ is a cocompact lattice in the product of automorphism groups of regular trees $\mathcal{T}_{i}$ of valency at least 3 and $G_{i}:=\overline{\operatorname{pr}_{i}(\Gamma)}$ acts transitively on the boundary $\mathcal{T}_{i}(\infty)$, from the fact that $\mathrm{H}_{\mathrm{cb}}^{2}\left(G_{i}\right)=0$ [9] we obtain that $\mathrm{H}_{\mathrm{b}}^{2}(\Gamma)=0$.

(2) If $\Gamma<\prod \mathbf{G}_{\alpha}\left(k_{\alpha}\right)$ is an irreducible lattice, where $\mathbf{G}_{\alpha}$ are $k_{\alpha}$-almost simple groups over local fields $k_{\alpha}$, with $\sum \operatorname{rank}_{k_{\alpha}} \mathbf{G}_{\alpha} \geqslant 2$, then $\mathrm{H}_{\mathrm{b}}^{2}(\Gamma)$ injects into $\mathrm{H}^{2}(\Gamma)$ [9], and both spaces are finite dimensional. In this case Margulis' superrigidity theorem applies and implies Corollary 1.6.

\section{On the proof and the organization of the paper}

The proof consists of two separate independent parts: roughly speaking, in the first we define an algebraic function which, via properties related to boundary maps, characterizes the representations up to conjugacy, while in the second we interpret this algebraic function in cohomological terms.

More specifically, we define the Hermitian triple product $\langle\cdot, \cdot, \cdot\rangle$ on triples of maximal totally isotropic subspaces in generic position, which corresponds in the case of $\mathrm{SU}(1, n)$ to the classical notion of Hermitian triple product [16]. Realizing $\mathrm{SU}(p, q)=: G$ as the real points of an algebraic group and considering the corresponding complexified Hermitian triple product $\langle\cdot, \cdot, \cdot\rangle_{\mathbb{C}}$, standard arguments in Zariski topology show that the Hermitian triple product can distinguish among representations when evaluated on the image of a $\Gamma$-equivariant measurable map $\varphi: B \rightarrow \mathrm{Is}_{\langle\cdot, \cdot\rangle}$, where $B$ is a double ergodic amenable Poisson boundary for $\Gamma$ and $\mathrm{Is}_{\langle\cdot, \cdot,\rangle}$ is the Grassmannian of maximal totally isotropic subspaces.

The second step consists in relating the pull-back, via $\varphi$, of the Hermitian triple product to the pull-back, via $\pi$, of the bounded Kähler class $\kappa_{G}^{\mathrm{b}}$. To do this, using the double ergodicity and once again the amenability of the $\Gamma$-space $B$, we identify as usual the second bounded cohomology group $\mathrm{H}_{\mathrm{b}}^{2}(\Gamma, \mathbb{R})$ of $\Gamma$ with the space $\mathcal{Z} L_{\text {alt }}^{\infty}\left(B^{3}\right)^{\Gamma}$ of essentially bounded alternating $\Gamma$-invariant cocycles on $B^{3}$ [9]. Moreover, we compute $\mathrm{H}_{\mathrm{cb}}^{2}(G, \mathbb{R})$ as the cohomology of an appropriate resolution on generic configurations of points in the Shilov boundary $\check{S}$ of $\mathcal{X}$; the class corresponding under the isomorphism to the bounded Kähler class has then a representative $\beta_{G}$ which is a strict Borel cocycle on generic triples of points in the Shilov boundary and hence the pull-back $\pi^{*}: \mathrm{H}_{\mathrm{cb}}^{2}(G, \mathbb{R}) \rightarrow \mathrm{H}_{\mathrm{b}}^{2}(\Gamma, \mathbb{R})$ can be implemented directly via the map $\varphi$ (after observing that $\mathrm{Is}_{\langle\cdot, \cdot\rangle} \simeq S$ ). Moreover, $\beta_{G}$ has the further crucial property that its exponential is essentially the Hermitian triple product which could distinguish among Zariski dense representations: the same property is hence inherited by the pull-back of the cohomology class of $\beta_{G}$ and thus by the pull-back of the bounded Kähler class.

The paper is organized as follows.

- In Section 3 we describe both the ball and the hyperboloid model for the Hermitian symmetric space associated to $G$, together with their explicit isomorphism which relates also the Shilov boundary in the ball model with the subset $\left.\mathrm{Is}_{\langle\cdot, .}\right\rangle$ of the boundary in the hyperboloid model. We also describe here the complexification of $G$ and $\operatorname{Is}_{\langle\cdot, \cdot\rangle}$. 
- In Section 4 we define the Hermitian triple product $\langle\cdot, \cdot, \cdot\rangle$, its complexified version $\langle\cdot, \cdot, \cdot\rangle_{\mathbb{C}}$, and describe their relation. Then we show the essential lemma that $\langle\cdot, \cdot, \cdot\rangle_{\mathbb{C}}$ is not constant if and only if $p \neq q$.

- In Section 5 we explicit the correspondence between generators of various cohomology groups in degree two (notably $\mathrm{H}^{2}(G, \mathbb{Z}), \mathrm{H}_{\mathrm{c}}^{2}(G, \mathbb{R})$, and $\mathrm{H}_{\mathrm{cb}}^{2}(G, \mathbb{R})$ ), and we define a cocycle $\beta_{G}$ on the Shilov boundary in the ball model of the Hermitian symmetric space, which extends the cocycle $c_{G}$ on the interior corresponding to the Kähler form (see 1.1).

- In Section 6 we define the resolution whose cohomology computes the bounded continuous cohomology of $G$ and we show that the cohomology class of the extension $\beta_{G}$ corresponds to the bounded Kähler class $\kappa_{G}^{\mathrm{b}}$ under the canonical isomorphism in cohomology.

- In Section 7 we construct the boundary map $\varphi: B \rightarrow \mathrm{Is}_{\langle\cdot, \cdot\rangle}$ into the space of maximal totally isotropic subspaces, by first recalling the existence of a Poisson boundary for $\Gamma$ which implies, by standard arguments using the amenability of the action of $\Gamma$ on $B$, the existence of a $\Gamma$-equivariant measurable map from $B$ into the space of probability measure on $G / P$, where $P$ is a minimal parabolic subgroup. Using the Zariski density of the representation and Furstenberg's boundary theory, we prove following [24] that the action of $\pi(\Gamma)$ on $G / P$ is mean proximal, which allows us to deduce that in fact the boundary map alluded above takes values into $G / P$. Composition with the projection $G / P \rightarrow G / Q \simeq \mathrm{Is}_{\langle\cdot, \cdot,\rangle}$, where $Q$ is an appropriate maximal parabolic containing $P$, gives the required map. Furthermore we prove that the images via $\varphi$ of almost all points in $B$ are pairwise in generic position.

- In Section 8, using the isomorphism (3.2) between the Shilov boundary and the space of maximal totally isotropic subspaces, we put to use the boundary map in Section 7 and the resolution in Section 5 to show that indeed the pull-back of the Kähler class is represented by the composition of $\varphi$ with the cocycle $\beta_{G}$ defined in Section 5 .

- In Section 9 we prove all results stated in the introduction.

\section{Preliminaries}

\subsection{Two models of Hermitian symmetric spaces}

Let $V$ be a complex vector space with a non-degenerate Hermitian form $\langle\cdot, \cdot\rangle$. We start by recalling two models for the symmetric space $\mathcal{X}$ associated to the special unitary group $\mathrm{SU}(V,\langle\cdot, \cdot\rangle)$ and the explicit realization of the identification between them.

Let $p$ be the index of the Hermitian form $\langle\cdot, \cdot\rangle$, that is the maximal dimension of a totally isotropic subspace; then $p=\min \left\{p_{+}, p_{-}\right\}$, where $p_{\varepsilon}$ is the maximal dimension of a subspace $W \subset V$ such that $\left.\langle\cdot, \cdot\rangle\right|_{W}$ is $\varepsilon$-definite, for $\varepsilon \in\{+,-\}$. Modulo a change of sign, we may assume that $p=p_{+} \leqslant p_{-}$. The hyperboloid model $\mathcal{X}^{h}$ of $\mathcal{X}$ is the open subset of the Grassmannian $\operatorname{Gr}_{p}(V)$ of $p$-planes in $V$ given by,

$$
\mathcal{X}^{h}:=\left\{L \in \operatorname{Gr}_{p}(V):\left.\langle\cdot, \cdot\rangle\right|_{L} \text { is positive definite }\right\}
$$

and its closure in $\operatorname{Gr}_{p}(V)$ is

$$
\overline{\mathcal{X}^{h}}=\left\{L \in \operatorname{Gr}_{p}(V):\left.\langle\cdot, \cdot\rangle\right|_{L} \text { is semi-positive definite }\right\} .
$$

To describe the realization of $\mathcal{X}$ as a bounded symmetric domain, fix a subspace $L_{+} \in \mathcal{X}^{h}$ with orthogonal complement $L_{-}$and, for $\varepsilon \in\{+,-\}$, let $\langle\cdot, \cdot\rangle_{\varepsilon}$ be the restriction to $L_{\varepsilon}$ of $\varepsilon\langle\cdot, \cdot\rangle$. It is easy to see that for any $L \in \overline{\mathcal{X}^{h}}$ the orthogonal projection $\left.\operatorname{pr}_{L_{+}}\right|_{L}: L \rightarrow L_{+}$is an isomorphism 
and hence we may define

$$
E(L):=\operatorname{pr}_{L_{-}} \circ\left(\left.\operatorname{pr}_{L_{+}}\right|_{L}\right)^{-1} \in \operatorname{Lin}\left(L_{+}, L_{-}\right),
$$

where $\operatorname{Lin}\left(L_{+}, L_{-}\right)$is the space of linear maps from $L_{+}$to $L_{-}$. Moreover, for all $L \in \overline{\mathcal{X}^{h}}, E(L)$ is semi-positive definite and hence the map

$$
E: \overline{\mathcal{X}^{h}} \rightarrow \operatorname{Lin}\left(L_{+}, L_{-}\right)
$$

defines an identification between $\overline{\mathcal{X}^{h}}$ and

$$
\overline{\mathcal{X}^{b}}:=\left\{A \in \operatorname{Lin}\left(L_{+}, L_{-}\right): I d_{+}-A^{*} A \text { is semi-positive definite }\right\},
$$

where $A^{*}$ is the adjoint map with respect to $\langle\cdot, \cdot\rangle_{\varepsilon}$. Furthermore, $E$ identifies $\mathcal{X}^{h}$ with

$$
\mathcal{X}^{b}:=\left\{A \in \operatorname{Lin}\left(L_{+}, L_{-}\right): I d_{+}-A^{*} A \text { is positive definite }\right\}
$$

(whose closure in $\operatorname{Lin}\left(L_{+}, L_{-}\right)$is $\overline{\mathcal{X}^{b}}$ ), and the space $\mathrm{Is}_{\langle\cdot, \cdot\rangle}$ of totally isotropic $p$-subspaces with the Shilov boundary

$$
\check{S}=\left\{A \in \operatorname{Lin}\left(L_{+}, L_{-}\right): I d_{+}-A^{*} A=0\right\},
$$

of the bounded symmetric domain $\mathcal{X}^{b}$.

\subsection{Complexification}

We turn now to the description of a suitable model of the complexification of $\mathrm{SU}(V,\langle\cdot, \cdot\rangle)$, and the space Is $\mathrm{I}_{\langle\cdot, \cdot\rangle}$. For this, fix a real structure $v \rightarrow \bar{v}$ on the $\mathbb{C}$-vector space $V$. Let $V_{\mathbb{C}}:=V \times V$ and define

$$
\begin{aligned}
\Delta_{V}: V & \rightarrow V_{\mathbb{C}} \\
v & \mapsto(v, \bar{v}) .
\end{aligned}
$$

Then

$$
\tau(v, w):=(\bar{w}, \bar{v})
$$

gives a real structure on $V_{\mathbb{C}}$ with fixed point set $\Delta_{V}(V)$. The form

$$
\begin{aligned}
{[\cdot, \cdot]: V \times V } & \rightarrow \mathbb{C} \\
(v, w) & \mapsto[v, w]:=\langle v, \bar{w}\rangle
\end{aligned}
$$

is $\mathbb{C}$-bilinear and non-degenerate and, with an appropriate choice of the real structure $v \rightarrow \bar{v}$, we may assume that it is symmetric.

Let $A=\mathbb{C} \times \mathbb{C}$ be the algebra, product of two copies of $\mathbb{C}$, with involution

$$
\begin{aligned}
\sigma: A & \rightarrow A \\
(\lambda, \mu) & \mapsto(\mu, \lambda),
\end{aligned}
$$

and define $\Delta_{\mathbb{C}}: \mathbb{C} \rightarrow \mathbb{C} \times \mathbb{C}$ by $\Delta_{\mathbb{C}}(\lambda)=(\lambda, \bar{\lambda})$. Then

$$
\tau(\lambda, \mu)=(\bar{\mu}, \bar{\lambda})
$$


defines a real structure on $A$ with fixed point set $\Delta_{\mathbb{C}}(\mathbb{C})$. Componentwise scalar multiplication gives an $A$-module structure on $V_{\mathbb{C}}$, defined over $\mathbb{R}$. The form

$$
\begin{aligned}
F: V_{\mathbb{C}} \times V_{\mathbb{C}} & \rightarrow A \\
\left(\left(v_{1}, w_{1}\right),\left(v_{2}, w_{2}\right)\right) & \mapsto\left(\left[v_{1}, w_{2}\right],\left[v_{2}, w_{1}\right]\right)
\end{aligned}
$$

enjoys then the following properties:

(1) $F$ is Hermitian symmetric, that is, for all $a, b \in A$ and $x, y \in V_{\mathbb{C}}$,

(a) $F(a x, b y)=a \sigma(b) F(x, y)$, and

(b) $F(y, x)=\sigma(F(x, y))$.

(2) $F$ is defined over $\mathbb{R}$ and extends the Hermitian form $\langle\cdot, \cdot\rangle$, namely

$$
F\left(\Delta_{V}(u), \Delta_{V}(v)\right)=\Delta_{\mathbb{C}}(\langle u, v\rangle)
$$

for all $u, v \in V$.

The group $\mathrm{GL}_{A}\left(V_{\mathbb{C}}\right)$ of $A$-module automorphisms of $V_{\mathbb{C}}$ is defined over $\mathbb{R}$ and so is the subgroup

$$
\mathbf{G}=\left\{T \in \mathrm{GL}_{A}\left(V_{\mathbb{C}}\right): \operatorname{det}_{A} T=1_{A}, T \text { preserves } F\right\},
$$

where $\operatorname{det}_{A}$ is the $A$-valued determinant. Identifying $\mathrm{GL}_{A}\left(V_{\mathbb{C}}\right)$ with $\mathrm{GL}(V) \times \mathrm{GL}(V)$, we get

$$
\mathbf{G}=\left\{\left(g, g^{b^{-1}}\right): g \in \mathrm{SL}(V)\right\}
$$

where $b$ denotes the adjoint with respect to the symmetric form $[\cdot, \cdot]$. Finally, restricting to $\Delta_{V}(V)$ the action of $\mathbf{G}(\mathbb{R})$ gives an identification of $\mathrm{SU}(V,\langle\cdot, \cdot\rangle)$ with $\mathbf{G}(\mathbb{R})$.

The set of free $A$-submodules of rank $p$ consisting of totally $F$-isotropic vectors may be identified with the projective variety

$$
\operatorname{Is}_{F}=\left\{(U, W) \in \operatorname{Gr}_{p}(V) \times \operatorname{Gr}_{p}(V):[u, w]=0, \text { for all } u \in U, w \in W\right\},
$$

which is defined over $\mathbb{R}$. The action of $\mathbf{G}$ on $\operatorname{Is}_{F}$ is also defined over $\mathbb{R}$, and the map

$$
\begin{aligned}
\Delta_{\mathrm{Is}_{\langle\cdot, \cdot\rangle}} \mathrm{Is}_{\langle\cdot, \cdot\rangle} & \rightarrow \operatorname{Is}_{F}(\mathbb{R}) \\
L & \mapsto(L, \bar{L})
\end{aligned}
$$

is an identification which is equivariant with respect to the identification $\mathrm{SU}(V,\langle\cdot, \cdot\rangle) \simeq \mathbf{G}(\mathbb{R})$.

The group $\mathrm{SU}(V,\langle\cdot, \cdot\rangle)$ acts transitively on $\mathrm{Is}_{\langle\cdot, \cdot\rangle}$ and Witt's theorem implies that it acts transitively on the set

$$
\mathrm{Is}_{\langle\cdot, \cdot\rangle}^{(2)}=\left\{\left(L_{1}, L_{2}\right) \in\left(\mathrm{Is}_{\langle\cdot, \cdot\rangle}\right)^{2}: L_{1} \cap L_{2}=\{0\}\right\}
$$

of pairs of transverse totally isotropic $p$-subspaces.

\section{The Hermitian triple product}

We proceed now to define an invariant for the action of $\mathrm{SU}(V,\langle\cdot, \cdot\rangle)$ on $\operatorname{Gr}_{p}(V)^{3}$. Given $L_{1}, L_{2} \in \operatorname{Gr}_{p}(V)$ and $B_{i}=\left\{b_{i}^{j}: 1 \leqslant j \leqslant p\right\}$ a basis of $L_{i}$, set

$$
\left\langle B_{1}, B_{2}\right\rangle:=\operatorname{det}\left(\left\langle b_{1}^{r}, b_{2}^{s}\right\rangle_{r, s}\right) .
$$


If $C_{i}$ is another basis of $L_{i}$, and $A_{i}$ is the matrix of change of basis from $B_{i}$ to $C_{i}$, we have

$$
\left\langle C_{1}, C_{2}\right\rangle=\operatorname{det} A_{1}\left\langle B_{1}, B_{2}\right\rangle \overline{\operatorname{det} A_{2}} .
$$

Given now $L_{1}, L_{2}, L_{3} \in \mathrm{Gr}_{p}(V)$, and $B_{i}, C_{i}$ bases of $L_{i}$, it follows from (4.1) that $\left\langle B_{1}, B_{2}\right\rangle$ $\left\langle B_{2}, B_{3}\right\rangle\left\langle B_{3}, B_{1}\right\rangle$ differs from $\left\langle C_{1}, C_{2}\right\rangle\left\langle C_{2}, C_{3}\right\rangle\left\langle C_{3}, C_{1}\right\rangle$ by a positive real, and hence we obtain a well defined invariant

$$
\left\langle L_{1}, L_{2}, L_{3}\right\rangle:=\left\langle B_{1}, B_{2}\right\rangle\left\langle B_{2}, B_{3}\right\rangle\left\langle B_{3}, B_{1}\right\rangle \in \mathbb{R}_{+}^{\times} \backslash \mathbb{C}
$$

which we call the Hermitian triple product (by analogy with [16, Section 2.2.5]).

Observe that if $L_{i} \in \overline{\mathcal{X}^{h}}$, we can write $L_{i}=\left\{v+E\left(L_{i}\right) v: v \in L_{+}\right\}$, so that, if $\left\{v_{1}, \ldots, v_{p}\right\}$ is an orthonormal basis of $L_{+}$, then $B_{i}=\left\{v_{k}+E\left(L_{i}\right) v_{k}: 1 \leqslant k \leqslant p\right\}$ is a basis of $L_{i}$. One can easily check that

$$
\left\langle B_{1}, B_{2}\right\rangle=\operatorname{det}\left(I d_{+}-E\left(L_{2}\right)^{*} E\left(L_{1}\right)\right),
$$

and hence one has in $\mathbb{R}_{+}^{\times} \backslash \mathbb{C}$ the following equality

$$
\left\langle L_{1}, L_{2}, L_{3}\right\rangle=\prod_{i=1}^{3} \operatorname{det}\left(I d_{+}-E\left(L_{i+1}\right)^{*} E\left(L_{i}\right)\right),
$$

where the indices are to be taken modulo 3 .

Observing that $L_{1} \cap L_{2}^{\perp}=\{0\}$ if and only if $\left\langle B_{1}, B_{2}\right\rangle \neq 0$ (where $B_{i}$ is a basis of $L_{i}$ ), we deduce that on the space $\mathrm{Is}_{\langle\cdot, \cdot\rangle}^{(3)}$ of triples of pairwise transverse maximal totally isotropic subspaces, the Hermitian triple product $\langle\cdot, \cdot, \cdot\rangle$ takes values in $\mathbb{R}_{+}^{\times} \backslash \mathbb{C}^{\times}$.

The set of values taken by the Hermitian triple product on the Shilov boundary shows the remarkable difference between the cases $p_{+}=p_{-}$and $p_{+} \neq p_{-}$, as recorded in the following:

LEMMA 4.1. - The set of values Val taken by the Hermitian triple product on $\mathrm{Is}_{\langle\cdot, \cdot,\rangle}^{(3)}$ is as follows:

(i) If $p_{+}=p_{-}$,

$$
\mathrm{Val}= \begin{cases}\{ \pm i\} \bmod \mathbb{R}_{+}^{\times} & \text {if } p \text { is odd } \\ \{ \pm 1\} \bmod \mathbb{R}_{+}^{\times} & \text {if } p \text { is even }\end{cases}
$$

(ii) If $p_{+}<p_{-}$,

$$
\mathrm{Val}= \begin{cases}\{z:|z|=1, \Re z \leqslant 0\} \bmod \mathbb{R}_{+}^{\times} & \text {if } p_{+}=1, \\ \mathbb{R}_{+}^{\times} \backslash \mathbb{C}^{\times} & \text {if } p_{+}>1 .\end{cases}
$$

Proof. - We say that

$$
A_{1}, A_{2} \in \check{S} \text { are transverse if } \operatorname{det}\left(I d_{+}-A_{2}^{*} A_{1}\right) \neq 0,
$$

and define $\delta: \check{S}^{3} \rightarrow \mathbb{C}$ by

$$
\delta\left(A_{1}, A_{2}, A_{3}\right):=\operatorname{det}\left(I d_{+}-A_{2}^{*} A_{1}\right) \operatorname{det}\left(I d_{+}-A_{3}^{*} A_{2}\right) \operatorname{det}\left(I d_{+}-A_{1}^{*} A_{3}\right) .
$$

(i) In this case we have that $\operatorname{dim} L_{+}=\operatorname{dim} L_{-}$and every $A \in \check{S}$ is an isomorphism of unitary spaces as it verifies $I d_{+}=A^{*} A$. In particular we have that $A A^{*}=I d_{-}$. Given now 
$A_{1}, A_{2}, A_{3} \in \check{S}$ pairwise transverse, set $C_{i}=A_{i+1}^{*} A_{i}$, for $i=1,2,3$ (with the indices intended modulo 3 ), so that

$$
C_{3} C_{2} C_{1}=I d_{+}
$$

Since $C_{i}$ is unitary, we have

$$
\overline{\operatorname{det}\left(I d_{+}-C_{i}\right)}=\operatorname{det}\left(I d_{+}-C_{i}^{-1}\right)=(-1)^{p} \operatorname{det}\left(C_{i}\right)^{-1} \operatorname{det}\left(I d_{+}-C_{i}\right),
$$

which implies, taking into account (4.6), that

$$
\overline{\delta\left(A_{1}, A_{2}, A_{3}\right)}=(-1)^{p} \delta\left(A_{1}, A_{2}, A_{3}\right) .
$$

This shows that $\delta\left(A_{1}, A_{2}, A_{3}\right)$ is purely imaginary if $p$ is odd and real if $p$ is even. Taking appropriate special matrices shows that the inclusions so obtained are in fact the equalities in (4.4).

(ii) In case $p_{+}=1$, we have the classical Hermitian triple product of isotropic vectors and the claim follows for instance from [16, Section 7.1].

In case $2 \leqslant p_{+}<p_{-}$, let $p=p_{+}$and $q=p_{-}$, for notational simplicity; by choosing orthonormal bases in $L_{+}, L_{-}$, we have the identifications $\operatorname{Lin}\left(L_{+}, L_{-}\right) \simeq M_{q, p}(\mathbb{C})$ and $\check{S} \simeq S_{p}:=\left\{A \in M_{q, p}(\mathbb{C}): A^{*} A=I_{p}\right\}$. Let

$$
X=\left(\begin{array}{c}
I_{p} \\
0
\end{array}\right), \quad Y=\left(\begin{array}{c}
-I_{p} \\
0
\end{array}\right), \quad \text { and } \quad Z=\left(\begin{array}{c}
Z_{1} \\
Z_{2}
\end{array}\right)
$$

where

$$
Z_{1}=\left(\begin{array}{cccc}
\lambda_{1} & 0 & \ldots & 0 \\
0 & \lambda_{2} & \ldots & 0 \\
\vdots & \vdots & \ddots & \vdots \\
0 & 0 & \ldots & \lambda_{p}
\end{array}\right) \text { and } Z_{2}=\left(\begin{array}{cccc}
\mu & 0 & \ldots & 0 \\
0 & 0 & \ldots & 0 \\
\vdots & \vdots & \ddots & \vdots \\
0 & 0 & \ldots & 0
\end{array}\right) \text {. }
$$

Then $X, Y \in S_{p}$; moreover $Z \in S_{p}$ if and only if $\left|\lambda_{1}\right|^{2}+|\mu|^{2}=1$, and $\left|\lambda_{i}\right|=1$ for $2 \leqslant i \leqslant p$. Furthermore, $X, Y, Z$ are pairwise transverse if and only if $\lambda_{i} \notin\{-1,1\}$, for $1 \leqslant i \leqslant p$, which we assume from now on. Then a computation gives

$$
\delta(X, Y, Z)=2^{2 p-1}\left(1-\left|\lambda_{1}\right|^{2}+\left(\lambda_{1}-\overline{\lambda_{1}}\right)\right) \prod_{j=2}^{p}\left(\lambda_{j}-\overline{\lambda_{j}}\right),
$$

which easily implies the claim.

Moving on to the complexified situation, let $\operatorname{Gr}_{p}^{A}(V)$ be the Grassmannian of free $A$ submodules of $V_{\mathbb{C}}$ of rank $p$. For $\mathcal{L}_{1}, \mathcal{L}_{2} \in \mathrm{Gr}_{p}^{A}(V)$, and $B_{i}=\left\{b_{i}^{j}: 1 \leqslant j \leqslant p\right\}$ a basis of $\mathcal{L}_{i}$ over $A$, set

$$
\left\langle B_{1}, B_{2}\right\rangle_{\mathbb{C}}:=\operatorname{det}_{A}\left(F\left(b_{1}^{r}, b_{2}^{s}\right)_{r, s}\right) \in A
$$

We say that for $i=1,2$

$$
\mathcal{L}_{i}=V_{i} \times W_{i} \text { are transverse if } V_{1} \cap{ }^{\perp} W_{2}=\{0\} \text { and } W_{1} \cap{ }^{\perp} V_{2}=\{0\},
$$


where ${ }^{\perp} U$ denotes the orthogonal of a subspace $U \subset V$ with respect to the symmetric form $[\cdot, \cdot]$. Then

$$
\left\langle B_{1}, B_{2}\right\rangle_{\mathbb{C}} \in A^{\times} \quad \text { if and only if } \quad \mathcal{L}_{1}, \mathcal{L}_{2} \text { are transverse. }
$$

If $C_{i}$ is another $A$-basis of $\mathcal{L}_{i}$ and $A_{i} \in M_{p, p}(A)$ is the change of basis from $B_{i}$ to $C_{i}$, we have

$$
\left(F\left(c_{1}^{r}, c_{2}^{s}\right)_{r, s}\right)=A_{1}\left(F\left(b_{1}^{r}, b_{2}^{s}\right)_{r, s}\right) \sigma\left(A_{2}^{t}\right)
$$

Setting

$$
\left\langle B_{1}, B_{2}, B_{3}\right\rangle_{\mathbb{C}}=\left\langle B_{1}, B_{2}\right\rangle_{\mathbb{C}}\left\langle B_{2}, B_{3}\right\rangle_{\mathbb{C}}\left\langle B_{3}, B_{1}\right\rangle_{\mathbb{C}}
$$

we deduce from (4.9) that

$$
\left\langle C_{1}, C_{2}, C_{3}\right\rangle_{\mathbb{C}}=N\left(\operatorname{det}_{A} A_{1}\right) N\left(\operatorname{det}_{A} A_{2}\right) N\left(\operatorname{det}_{A} A_{3}\right)\left\langle B_{1}, B_{2}, B_{3}\right\rangle_{\mathbb{C}},
$$

where $N: A \rightarrow \mathbb{C}$ is the norm map given by $N(a) 1_{A}=a \sigma(a)$. Thus, for any $\mathcal{L}_{1}, \mathcal{L}_{2}, \mathcal{L}_{3} \in$ $\operatorname{Gr}_{p}^{A}(V)$, we obtain a well defined G-invariant Hermitian triple product

$$
\left\langle\mathcal{L}_{1}, \mathcal{L}_{2}, \mathcal{L}_{3}\right\rangle_{\mathbb{C}}=\left\langle B_{1}, B_{2}\right\rangle_{\mathbb{C}}\left\langle B_{2}, B_{3}\right\rangle_{\mathbb{C}}\left\langle B_{3}, B_{1}\right\rangle_{\mathbb{C}} \in \mathbb{C}^{\times} \backslash A
$$

LEMMA 4.2.-

(i) $\mathbf{G}$ acts transitively on $\mathrm{Is}_{F}$;

(ii) For every $\mathcal{L} \in \mathrm{Is}_{F}$, the set

$$
\operatorname{nt}(\mathcal{L}):=\left\{\mathcal{L}^{\prime} \in \operatorname{Is}_{F}: \mathcal{L}^{\prime} \text { is not transverse to } \mathcal{L}\right\}
$$

is a proper Zariski closed subset of $\operatorname{Is}_{F}$.

Proof. - (i) Choose a basis $\left\{e_{1}, \ldots, e_{n}\right\}$ of $V$ such that $\left[e_{i}, e_{j}\right]=\delta_{i j}$, and define

$$
V_{0}=\mathbb{C} e_{1}+\cdots+\mathbb{C} e_{p} \text { and } \quad W_{0}=\mathbb{C} e_{p+1}+\cdots+\mathbb{C} e_{2 p} .
$$

Since ${ }^{\perp} V_{0}=\mathbb{C} e_{p+1}+\cdots+\mathbb{C} e_{n}$, we have that $\left(V_{0}, W_{0}\right) \in \mathrm{Is}_{F}$. Let $(U, W) \in \mathrm{Is}_{F}$ and take $g \in \mathrm{SL}(V)$ such that $g U=V_{0}$. Then $g^{*-1}(W) \subset{ }^{\perp} V_{0}$. Now,

$$
\operatorname{Stab}_{\mathrm{SL}(V)}\left(V_{0}\right)=\left\{\left(\begin{array}{cc}
A & B \\
0 & C
\end{array}\right): \operatorname{det} A \operatorname{det} C=1\right\},
$$

and hence the action of $\operatorname{Stab}_{\mathrm{SL}(V)}\left(V_{0}\right)$ on ${ }^{\perp} V_{0}$ via $h \rightarrow h^{*-1}$ gives the full $\mathrm{GL}\left({ }^{\perp} V_{0}\right)$-action on ${ }^{\perp} V_{0}$; since $\operatorname{dim} g^{*-1}(W)=\operatorname{dim} W_{0}$, there is $h \in \operatorname{Stab}_{\mathrm{SL}(V)}\left(V_{0}\right)$ with $h^{*-1}\left(g^{*-1}(W)\right)=W_{0}$, and thus $\left(h g, h g^{*-1}\right)(U, W)=\left(V_{0}, W_{0}\right)$.

(ii) follows from (4.8).

Remark 4.3. - One can show that $\mathbf{G}$ acts transitively on the set of pairs of transverse elements in $\mathrm{Is}_{F}$, but we shall not need this fact.

The set

$$
\mathrm{Is}_{F}^{(3)}=\left\{\left(\mathcal{L}_{1}, \mathcal{L}_{2}, \mathcal{L}_{3}\right) \in\left(\mathrm{Is}_{F}\right)^{3}: \mathcal{L}_{1}, \mathcal{L}_{2}, \mathcal{L}_{3} \text { are pairwise transverse }\right\}
$$


is a Zariski open subset of $\left(\operatorname{Is}_{F}\right)^{3}$, defined over $\mathbb{R}$. The Cartesian product of the identification $\Delta_{\mathrm{Is}_{\langle\cdot, \cdot\rangle}}$ in (3.4) induces an identification

$$
\Delta_{\mathrm{Is}\langle\cdot, \cdot\rangle}^{(3)}: \operatorname{Is}_{\langle\cdot, \cdot\rangle}^{(3)} \rightarrow \operatorname{Is}_{F}^{(3)}(\mathbb{R}) .
$$

The affine space $A$ with its $\mathbb{R}$-structure given by $\tau$ (see (3.3)) is acted upon by $\mathbb{C}^{\times}$, and this action is defined over $\mathbb{R}$; we denote by $\mathbb{P}^{1}(\mathbb{C})$ the quotient $\mathbb{C}^{\times} \backslash(A \backslash\{0\})$ with its corresponding $\mathbb{R}$-structure. Then the Hermitian triple product gives a regular $\mathbf{G}$-invariant map

$$
\langle\cdot, \cdot, \cdot\rangle_{\mathbb{C}}: \operatorname{Is}_{F}^{(3)} \rightarrow \mathbb{P}^{1}(\mathbb{C})
$$

defined over $\mathbb{R}$ and with image contained in $\mathbb{C}^{\times} \backslash A^{\times}$. The map $\Delta_{\mathbb{C}}$ giving the real structure on $A$ induces a map

$$
\bar{\Delta}: \mathbb{R}_{+}^{\times} \backslash \mathbb{C}^{\times} \rightarrow \mathbb{C}^{\times} \backslash A^{\times},
$$

which is also a group homomorphism with kernel of order 2 , and one verifies that the following diagram

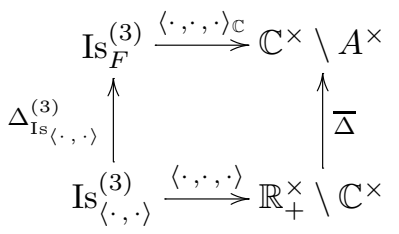

commutes.

For $\mathcal{L}_{1}, \mathcal{L}_{2} \in \mathrm{Is}_{F}(\mathbb{R})$ transverse, let $\mathcal{O}_{\mathcal{L}_{1}, \mathcal{L}_{2}} \subset \mathrm{Is}_{F}$ be the Zariski open, connected subset of those elements $\mathcal{L} \in \mathrm{Is}_{F}$ which are transverse to $\mathcal{L}_{1}$ and $\mathcal{L}_{2}$. Then the function

$$
P_{\mathcal{L}_{1}, \mathcal{L}_{2}}: \mathcal{O}_{\mathcal{L}_{1}, \mathcal{L}_{2}} \rightarrow \mathbb{C}^{\times} \backslash A^{\times}
$$

given by $P_{\mathcal{L}_{1}, \mathcal{L}_{2}}(\mathcal{L}):=\left\langle\mathcal{L}_{1}, \mathcal{L}_{2}, \mathcal{L}\right\rangle_{\mathbb{C}}$ is regular and:

LEMMA 4.4. - If $p_{-}>p_{+}$, then $P_{\mathcal{L}_{1}, \mathcal{L}_{2}}^{m}$ is not constant for any $m \in \mathbb{Z}, m \neq 0$.

Here $P_{\mathcal{L}_{1}, \mathcal{L}_{2}}^{m}(\mathcal{L})=\left(P_{\mathcal{L}_{1}, \mathcal{L}_{2}}(\mathcal{L})\right)^{m}$, where the product is taken in $\mathbb{C}^{\times} \backslash A^{\times}$.

Proof. - Since $\mathbf{G}(\mathbb{R})$ is transitive on $\operatorname{Is}_{F}^{(2)}(\mathbb{R}) \simeq \operatorname{Is}_{\langle\cdot, \cdot\rangle}^{(2)}$ (see (3.4), (3.5), and (4.10)) we have $P_{\mathcal{L}_{1}, \mathcal{L}_{2}}\left(\mathcal{O}_{\mathcal{L}_{1}, \mathcal{L}_{2}}(\mathbb{R})\right)=\operatorname{Image}\left(\left.\langle\cdot, \cdot, \cdot\rangle_{\mathbb{C}}\right|_{\mathrm{Is}_{F}^{(3)}(\mathbb{R})}\right)$. When $p_{-}>p_{+}$, the latter is infinite by Lemma 4.1.

\section{The bounded Kähler class}

\subsection{The Dupont cocycle as an integral cohomology class}

Let $G$ be a connected simple Lie group with finite center and $\mathcal{X}$ its associated symmetric space. We assume that $\mathcal{X}$ is Hermitian symmetric, that is $\mathcal{X}$ carries a $G$-invariant complex structure. Fix $x_{0} \in \mathcal{X}$ a basepoint, let $K=\operatorname{Stab}_{G}\left(x_{0}\right)$, and let $\mathfrak{g}=\mathfrak{k} \oplus \mathfrak{p}$ be the corresponding Cartan decomposition of the Lie algebra $\mathfrak{g}$ of $G$. We shall equip $\mathcal{X}$ with the metric defined by $\frac{1}{2} B_{\mathfrak{g}}$, 
where $B_{\mathfrak{g}}$ is the Killing form of $\mathfrak{g}$. For the associated Kähler form $\omega$ we have then,

$$
\omega_{x_{0}}(X, Y)=\frac{1}{2} B_{\mathfrak{g}}(X, J Y), \quad \text { for all } X, Y \in \mathfrak{p},
$$

where $J \in \operatorname{End}(\mathfrak{p})$ is the complex structure obtained from the identification of $T_{x_{0}} \mathcal{X}$ with $\mathfrak{p}$. The Kähler form $\omega$ gives rise to the Dupont cocycle

$$
c_{G}\left(g_{1}, g_{2}, g_{3}\right):=\int_{\Delta\left(g_{1} x_{0}, g_{2} x_{0}, g_{3} x_{0}\right)} \omega,
$$

where for any three points $x, y, z \in \mathcal{X}, \Delta(x, y, z)$ denotes a 2 -simplex with vertices $x, y, z$ and geodesic sides. The (homogeneous) cocycle $c_{G}$ is $G$-invariant, differentiable and bounded [12] and thus defines both a continuous cohomology class $\kappa_{G} \in \mathrm{H}_{\mathrm{c}}^{2}(G, \mathbb{R})$ and a bounded continuous class $\kappa_{G}^{\mathrm{b}} \in \mathrm{H}_{\mathrm{cb}}^{2}(G, \mathbb{R})$ which correspond to each other via the canonical isomorphism $\mathrm{H}_{\mathrm{cb}}^{2}(G, \mathbb{R}) \simeq \mathrm{H}_{\mathrm{c}}^{2}(G, \mathbb{R})$ [9]. Taking into account that $\mathrm{H}_{\mathrm{c}}^{2}(G, \mathbb{R}) \simeq \mathbb{R}$, these classes are generators of their corresponding cohomology groups.

Denoting by $\mathrm{H}^{2}(G, \mathbb{R})$ (respectively $\mathrm{H}^{2}(G, \mathbb{Z})$ ) the Borel cohomology of $G$ with coefficients in $\mathbb{R}$ (respectively $\mathbb{Z}$ ), it is important for us in the sequel to determine the specific multiple of $c_{G}$ which is the image of a generator of $\mathrm{H}^{2}(G, \mathbb{Z}) \simeq \mathbb{Z}$ (see [19]) via the map $\mathrm{H}^{2}(G, \mathbb{Z}) \rightarrow \mathrm{H}^{2}(G, \mathbb{R})$. To this end, let $Z_{0} \in Z(\mathfrak{k})$ be the uniquely defined element in the center of $\mathfrak{k}$ such that $\left.\operatorname{ad}_{\mathfrak{g}}\left(Z_{0}\right)\right|_{\mathfrak{p}}=J, u: K \rightarrow \mathbb{T}$ a generator of the group $\operatorname{Hom}_{\mathrm{c}}(K, \mathbb{T})$ of continuous homomorphisms of $K$ into the circle $\mathbb{T}$, and $D u_{e}: \mathfrak{k} \rightarrow i \mathbb{R}$ its derivative at the identity.

LEMMA 5.1. - The cocycle

$$
c_{G}^{\mathbb{Z}}\left(g_{1}, g_{2}, g_{3}\right):=\frac{1}{2 \pi i} \frac{D u_{e}\left(Z_{0}\right)}{\operatorname{dim} \mathfrak{p}} c_{G}\left(g_{1}, g_{2}, g_{3}\right)
$$

determines a generator of $\mathrm{H}^{2}(G, \mathbb{Z})$.

Proof. - According to [13], such a cocycle $c_{G}^{\mathbb{Z}}$ can be represented by

$$
c_{G}^{\mathbb{Z}}\left(g_{1}, g_{2}, g_{3}\right)=\int_{\Delta\left(g_{1} x_{0}, g_{2} x_{0}, g_{3} x_{0}\right)} \Omega,
$$

where $\Omega$ is the invariant 2 -form on $\mathcal{X}$ whose value at $T_{x_{0}} \mathcal{X} \simeq \mathfrak{p}$ is

$$
\Omega_{x_{0}}(X, Y)=\frac{1}{4 \pi i} D u_{e}([X, Y]), \quad X, Y \in \mathfrak{p} .
$$

By using the decomposition $\mathfrak{k}=\mathbb{R} Z_{0} \oplus[\mathfrak{k}, \mathfrak{k}]$, we define a 2 -form $\omega_{1}$ on $\mathfrak{p}$ by the equation

$$
[X, Y]=\omega_{1}(X, Y) Z_{0}+C, \quad C \in[\mathfrak{k}, \mathfrak{k}] .
$$

Thus $D u_{e}([X, Y])=\omega_{1}(X, Y) D u_{e}\left(Z_{0}\right)$ and we proceed to relate $\omega_{1}$ to $\omega_{x_{0}}$. We have

$$
2 \omega_{x_{0}}(X, Y)=\operatorname{Tr}\left(\operatorname{ad}_{\mathfrak{g}}(X) \operatorname{ad}_{\mathfrak{g}}(J Y)\right)=\operatorname{Tr}\left(\operatorname{ad}_{\mathfrak{g}}(X) \operatorname{ad}_{\mathfrak{g}}\left(\left[Z_{0}, Y\right]\right)\right) .
$$

By expanding $\operatorname{ad}_{\mathfrak{g}}\left(\left[Z_{0}, Y\right]\right)$, and using (5.4), we obtain 


$$
\begin{aligned}
2 \omega_{x_{0}}(X, Y) & =-\operatorname{Tr}\left(\operatorname{ad}_{\mathfrak{g}}([X, Y]) \operatorname{ad}_{\mathfrak{g}}\left(Z_{0}\right)\right) \\
& =-\operatorname{Tr}\left(\omega_{1}(X, Y)\left(\operatorname{ad}_{\mathfrak{g}}\left(Z_{0}\right)\right)^{2}\right)-\operatorname{Tr}\left(\operatorname{ad}_{\mathfrak{g}}(C) \operatorname{ad}_{\mathfrak{g}}\left(Z_{0}\right)\right) \\
& =\operatorname{dim} \mathfrak{p} \omega_{1}(X, Y)-\operatorname{Tr}\left(\operatorname{ad}_{\mathfrak{g}}(C) \operatorname{ad}_{\mathfrak{g}}\left(Z_{0}\right)\right) .
\end{aligned}
$$

Using that $C \in[\mathfrak{k}, \mathfrak{k}]$, one checks that $\operatorname{Tr}\left(\operatorname{ad}_{\mathfrak{g}}(C) \operatorname{ad}_{\mathfrak{g}}\left(Z_{0}\right)\right)=0$, and thus

$$
D u_{e}([X, Y])=\frac{2}{\operatorname{dim} \mathfrak{p}} D u_{e}\left(Z_{0}\right) \omega_{x_{0}}(X, Y),
$$

which, together with (5.3) and (5.2), implies the lemma.

LEMMA 5.2.-

(i) If $G=\mathrm{SU}(p, q)$ then $c_{G}^{\mathbb{Z}}=\frac{1}{4 \pi(p+q)} c_{G}$;

(ii) If $G=\operatorname{PSU}(p, q)$ then $c_{G}^{\mathbb{Z}}=\frac{1}{4 \pi \operatorname{gcd}(p, q)} c_{G}$.

Proof. - The Lie algebra of $\mathrm{SU}(p, q)$ is

$$
\mathfrak{s u}(p, q)=\left\{X \in M_{p+q}(\mathbb{C}): \bar{X}^{t} H+H X=0\right\}, \quad \text { where } H=\left(\begin{array}{cc}
-I_{q} & 0 \\
0 & I_{p}
\end{array}\right) .
$$

For the Cartan involution $\theta(X)=-\bar{X}^{t}$, we have

$$
\mathfrak{p}=\left\{\left(\begin{array}{cc}
0 & B \\
\bar{B}^{t} & 0
\end{array}\right): B \in M_{q, p}(\mathbb{C})\right\} .
$$

For the complex structure on $\mathfrak{p}$ given by $B \rightarrow i B$ we obtain

$$
Z_{0}=\left(\begin{array}{cc}
\frac{i p}{p+q} I_{q} & 0 \\
0 & \frac{-i q}{p+q} I_{p}
\end{array}\right)
$$

If $G=\mathrm{SU}(p, q)$, then $K=\mathrm{S}(\mathrm{U}(q) \times \mathrm{U}(p))$, and a generator $u$ of $\operatorname{Hom}_{\mathrm{c}}(K, \mathbb{T})$ is given by

$$
u\left(\begin{array}{cc}
A & 0 \\
0 & B
\end{array}\right):=\operatorname{det} A .
$$

From this we deduce readily that

$$
D u_{e}\left(Z_{0}\right)=\frac{i p q}{p+q}
$$

which, together with $\operatorname{dim} \mathfrak{p}=2 p q$, implies (i).

For part (ii), if we let $G_{a}$ be the adjoint group $\operatorname{PSU}(p, q)$, then its maximal compact subgroup $K_{a}$ is the quotient of $K$ by

$$
Z(G)=\left\{\lambda I_{p+q}: \lambda^{p+q}=1\right\}
$$

and a generator $u_{a}$ of $\operatorname{Hom}\left(K_{a}, \mathbb{T}\right)$ is given by $u_{a}:=u^{n}$, where $n \in \mathbb{N}$ is minimal such that $\left.u^{n}\right|_{Z(G)}=1$. A computation gives then

$$
n=\frac{p+q}{\operatorname{gcd}(p, q)}
$$

which implies (ii). 


\subsection{Extension to the boundary}

Turning now to the ball model $\mathcal{X}^{b}$ of the symmetric space associated to $G=\mathrm{SU}(V,\langle\cdot, \cdot\rangle)$, we are going to extend the cocycle

$$
c(x, y, z):=\int_{\Delta(x, y, z)} \omega
$$

to (a subset of) the closure $\overline{\mathcal{X}^{b}} \subset \operatorname{Lin}\left(L_{+}, L_{-}\right)$. To this end, a formula for (5.5) due to Domic and Toledo will be useful. For $X, Y \in \overline{\mathcal{X}^{b}}$ transverse - that is $\operatorname{det}\left(I d_{+}-X^{*} Y\right) \neq 0$ (see (4.5)) - let

$$
\alpha_{G}(X, Y):=-2 \sum_{j=1}^{p} \arg \left(1-\lambda_{j}\right),
$$

where $\lambda_{1}, \ldots, \lambda_{p}$ are the eigenvalues of $X^{*} Y$ counted with multiplicity. Since $\left|\lambda_{j}\right| \leqslant 1$ and $\lambda_{j} \neq 1$, then $\arg \left(1-\lambda_{j}\right) \in[-\pi / 2, \pi / 2]$ is well defined.

For $X, Y, Z \in \overline{\mathcal{X}^{b}}$ pairwise transverse, let

$$
\beta_{G}(X, Y, Z)=\alpha_{G}(X, Y)+\alpha_{G}(Y, Z)+\alpha_{G}(Z, X) .
$$

Then

LEMMA 5.3 [11]. - For every $X, Y, Z \in \mathcal{X}^{b}$

$$
\beta_{G}(X, Y, Z)=\int_{\Delta(X, Y, Z)} \omega .
$$

Let $\overline{\mathcal{X}}^{(3)}$ denote the set of triples of pairwise transverse elements of $\mathcal{X}^{b}$. Then

LEMma 5.4. - The function $\beta_{G}:{\overline{\mathcal{X}^{b}}}^{(3)} \rightarrow[-\pi p, \pi p]$ is continuous, alternating, G-invariant, and $d \beta_{G}\left(X_{1}, X_{2}, X_{3}, X_{4}\right)=0$ for all 4 -tuples of pairwise transverse elements.

Proof. - The function $\beta_{G}$ is clearly continuous on $\overline{\mathcal{X}}^{(3)}$ and, by Lemma 5.3, satisfies all above properties on $\left(\mathcal{X}^{b}\right)^{3}$. Since $\left(\mathcal{X}^{b}\right)^{3}$ is dense in $\overline{\mathcal{X}^{b}}{ }^{(3)}$, we obtain the lemma.

Finally, it follows from the above formulæ that

$$
\mathrm{e}^{\frac{i}{2} \beta_{G}(X, Y, Z)}=\operatorname{det}\left(I d_{+}-Y^{*} X\right) \operatorname{det}\left(I d_{+}-Z^{*} Y\right) \operatorname{det}\left(I_{L_{+}}-X^{*} Z\right) \bmod \mathbb{R}^{+\times},
$$

for all $(X, Y, Z) \in{\overline{\mathcal{X}^{b}}}^{(3)}$. Taking into account (4.3), we obtain

$$
\mathrm{e}^{\frac{i}{2} \beta_{G}\left(E\left(L_{1}\right), E\left(L_{2}\right), E\left(L_{3}\right)\right)}=\left\langle L_{1}, L_{2}, L_{3}\right\rangle \quad \text { in } \mathbb{R}_{+}^{\times} \backslash \mathbb{C}^{\times}
$$

for all pairwise transverse $L_{1}, L_{2}, L_{3} \in \overline{\mathcal{X}^{h}}$.

\section{A resolution on the Shilov boundary}

Let $G=\mathrm{SU}(V,\langle\cdot, \cdot\rangle)$, let $\check{S}$ be the Shilov boundary of $\mathcal{X}^{b}$, and let us define, for every $n \geqslant 2$ and in analogy with the notation above,

$$
\check{S}^{(n)}=\left\{\left(X_{1}, X_{2}, \ldots, X_{n}\right) \in \check{S}^{n}: X_{i}, X_{j} \text { are transverse for all } i \neq j\right\} .
$$


For every $n \geqslant 2$, the Banach space $\mathcal{B}_{\text {alt }}^{\infty}\left(\check{S}^{(n)}\right)$ of bounded alternating Borel functions on $\check{S}^{(n)}$, equipped with the supremum norm, admits a natural coboundary operator

$$
d_{n}: \mathcal{B}_{\mathrm{alt}}^{\infty}\left(\check{S}^{(n)}\right) \rightarrow \mathcal{B}_{\mathrm{alt}}^{\infty}\left(\check{S}^{(n+1)}\right)
$$

defined in the usual way. The group $G$ acts by homeomorphisms of $\check{S}^{(n)}$ and hence isometrically on $\mathcal{B}_{\text {alt }}^{\infty}\left(\check{S}^{(n)}\right)$. Then, in the terminology of [9] and [27], we have:

LEMMA 6.1. - The complex

$$
0 \longrightarrow \mathbb{R} \stackrel{d_{0}}{\longrightarrow} \mathcal{B}^{\infty}(\check{S}) \stackrel{d_{1}}{\longrightarrow} \mathcal{B}_{\text {alt }}^{\infty}\left(\check{S}^{(2)}\right) \stackrel{d_{2}}{\longrightarrow} \cdots
$$

is a strong G-resolution.

Proof. - Let $\mathcal{B}_{\text {alt }}^{\infty}\left(\check{S}^{n}\right)$ be the space of alternating bounded Borel functions on $\check{S}^{n}$ (with the supremum norm $), d_{n}^{\prime}: \mathcal{B}_{\text {alt }}^{\infty}\left(\check{S}^{n}\right) \rightarrow \mathcal{B}_{\text {alt }}^{\infty}\left(\check{S}^{n+1}\right)$ the natural coboundary operator, $\mu$ a $G$-quasiinvariant probability measure on $\check{S}$ (for example, the $K$-invariant one), and

$$
\begin{aligned}
h_{n-1}^{\prime}: \mathcal{B}_{\mathrm{alt}}^{\infty}\left(\check{S}^{n}\right) & \rightarrow \mathcal{B}_{\mathrm{alt}}^{\infty}\left(\check{S}^{n-1}\right) \\
\alpha & \mapsto h_{n-1}^{\prime} \alpha
\end{aligned}
$$

where

$$
h_{n-1}^{\prime} \alpha\left(X_{2}, \ldots, X_{n}\right):=\int_{\check{S}} \alpha\left(X_{1}, X_{2}, \ldots, X_{n}\right) d \mu\left(X_{1}\right) .
$$

We have shown in [6] that the complex $\left(\mathcal{B}_{\text {alt }}^{\infty}\left(\check{S}^{\bullet}\right), d_{\bullet}\right)$ is a strong $G$-resolution of $\mathbb{R}$ with homotopy operators $h_{n}^{\prime}$. Let $r_{n}: \mathcal{B}_{\text {alt }}^{\infty}\left(\check{S}^{n}\right) \rightarrow \mathcal{B}_{\text {alt }}^{\infty}\left(\check{S}^{(n)}\right)$ denote the operator obtained by restricting functions to $\check{S}^{(n)}$ and $i_{n}: \mathcal{B}_{\text {alt }}^{\infty}\left(\check{S}^{(n)}\right) \rightarrow \mathcal{B}_{\text {alt }}^{\infty}\left(\check{S}^{n}\right)$ the one obtained by extending functions from $\check{S}^{(n)}$ to $\check{S}^{n}$ by setting them equal to zero on $\check{S}^{n} \backslash \check{S}^{(n)}$. Both $r_{n}$ and $i_{n}$ are $G$-equivariant; $r_{n}$ is norm decreasing and $i_{n}$ is norm preserving, thus they preserve the corresponding subspaces of $G$-continuous vectors. We have the simple relation

$$
d_{n}=r_{n+1} d_{n}^{\prime} i_{n}
$$

Observe that, while $r_{\bullet}$ is a morphism of complexes, $\left\{i_{n}\right\}_{n}$ fails to be. Define now

$$
h_{n}:=r_{n} h_{n}^{\prime} i_{n+1} \text {. }
$$

Since $h_{n}^{\prime}$ sends continuous vectors to continuous vectors, it follows from the above remarks that $h_{n}$ sends the subspace of continuous vectors in $\mathcal{B}_{\text {alt }}^{\infty}\left(\check{S}^{(n+1)}\right)$ into the subspace of continuous vectors in $\mathcal{B}_{\text {alt }}^{\infty}\left(\check{S}^{(n)}\right)$. All there remains to verify is that the $h_{n}$ 's are homotopy operators. Using the above definition of $h_{n}$ and the fact that $r_{\bullet}$ is a morphism of complexes, we have

$$
d_{n-1} h_{n-1}=d_{n-1} r_{n-1} h_{n-1}^{\prime} i_{n}=r_{n} d_{n-1}^{\prime} h_{n-1}^{\prime} i_{n},
$$

and

$$
h_{n} d_{n}=\left(r_{n} h_{n}^{\prime} i_{n+1}\right)\left(r_{n+1} d_{n}^{\prime} i_{n}\right) .
$$


Let $\alpha \in \mathcal{B}_{\text {alt }}^{\infty}\left(\check{S}^{n+1}\right)$ and define $\alpha^{\prime}:=i_{n+1} r_{n+1} \alpha$. Then

$$
\alpha^{\prime}\left(X_{1}, \ldots, X_{n+1}\right)= \begin{cases}\alpha\left(X_{1}, \ldots, X_{n+1}\right) & \text { if }\left(X_{1}, \ldots, X_{n+1}\right) \in \check{S}^{(n+1)}, \\ 0 & \text { otherwise. }\end{cases}
$$

Observe that if $\left(X_{2}, \ldots, X_{n+1}\right) \in \check{S}^{(n)}$, then

$$
\mu\left\{X_{1} \in \check{S}:\left(X_{1}, \ldots, X_{n+1}\right) \notin \check{S}^{(n+1)}\right\}=0
$$

and hence, for all $\left(X_{2}, \ldots, X_{n+1}\right) \in \check{S}^{(n)}$,

$$
\int_{\breve{S}} \alpha^{\prime}\left(X_{1}, X_{2}, \ldots, X_{n+1}\right) d \mu\left(X_{1}\right)=\int_{\check{S}} \alpha\left(X_{1}, X_{2}, \ldots, X_{n+1}\right) d \mu\left(X_{1}\right) .
$$

This implies that $r_{n} h_{n}^{\prime} i_{n+1} r_{n+1}=r_{n} h_{n}^{\prime}$ which, together with (6.1), shows that

$$
h_{n+1} d_{n+1}=r_{n} h_{n}^{\prime} d_{n}^{\prime} i_{n},
$$

and hence

$$
d_{n-1} h_{n-1}+h_{n} d_{n}=r_{n}\left(d_{n-1}^{\prime} h_{n-1}^{\prime}+h_{n}^{\prime} d_{n}^{\prime}\right) i_{n}=r_{n} i_{n}=I d_{\mathcal{B}_{\mathrm{alt}}^{\infty}\left(\check{S}^{(n)}\right)} .
$$

This concludes the proof of the lemma.

Applying now [9, Proposition 1.5.2] we obtain a canonical map

$$
\mathrm{H}^{\bullet}\left(\mathcal{B}_{\text {alt }}^{\infty}\left(\check{S}^{(\bullet)}\right)^{G}\right) \rightarrow \mathrm{H}_{\mathrm{cb}}^{\bullet}(G, \mathbb{R})
$$

from the cohomology of the complex $\left(\mathcal{B}_{\text {alt }}^{\infty}\left(\check{S}^{(\bullet)}\right)^{G}, d_{\bullet}\right)$ into the bounded continuous cohomology of $G$. In particular the function considered in Section 5.2

$$
\beta_{G}: \check{S}^{(3)} \rightarrow[-\pi p, \pi p]
$$

is a bounded, alternating, $G$-invariant cocycle on $\check{S}^{(3)}$ (see Lemma 5.4), and thus defines a class $\left[\beta_{G}\right] \in \mathrm{H}^{2}\left(\mathcal{B}_{\text {alt }}^{\infty}\left(\check{S}^{(\bullet)}\right)^{G}\right)$.

LEMMA 6.2. - Under the map (6.2), the class $\left[\beta_{G}\right]$ corresponds to the class $\kappa_{G}^{\mathrm{b}} \in \mathrm{H}_{\mathrm{cb}}^{2}(G, \mathbb{R})$.

Proof. - We consider the morphisms of complexes

$$
\mathcal{B}_{\mathrm{alt}}^{\infty}\left(\check{S}^{(\bullet)}\right) \rightarrow L^{\infty}\left(\check{S}^{\bullet}\right) \rightarrow L^{\infty}\left(G^{\bullet}\right)
$$

where the first one is obtained by considering a function on $\check{S}^{(n)}$ as a class in $L^{\infty}\left(\check{S}^{n}\right)$ (recall that $\breve{S}^{n} \backslash \breve{S}^{(n)}$ is a null set) and the second is obtained by realizing $\breve{S}$ as a homogeneous space of $G$ by means of choosing a basepoint $b \in \check{S}$. The composed morphism

$$
\mathcal{B}_{\text {alt }}^{\infty}\left(\check{S}^{(\bullet)}\right) \rightarrow L^{\infty}\left(G^{\bullet}\right)
$$

extends the identity and it follows then from Lemma 6.1 and [9, Proposition 1.5.2] that it implements the canonical map (6.2) in cohomology. What is left to be shown is that

$$
\bar{c}\left(g_{1}, g_{2}, g_{3}\right):=\beta_{G}\left(g_{1} b, g_{2} b, g_{3} b\right)
$$


is cohomologous to $c_{G}$ in $L^{\infty}\left(G^{\bullet}\right)$. Define for all 4 -tuples $t_{1}, t_{2}, t_{3}, t_{4}$ of pairwise transverse elements in $\overline{\mathcal{X}^{b}}$

$$
\square\left(t_{1}, t_{2}, t_{3}, t_{4}\right):=\beta_{G}\left(t_{1}, t_{2}, t_{3}\right)+\beta_{G}\left(t_{3}, t_{4}, t_{1}\right) .
$$

When $t_{i} \in \mathcal{X}^{b}$, this coincides with the integral of the Kähler class $\omega$ over an oriented quadrilateral with vertices $t_{1}, t_{2}, t_{3}, t_{4}$ and geodesic sides. Given now two triangles in $\mathcal{X}^{b}$ with vertices $x_{1}, x_{2}, x_{3}$ and $y_{1}, y_{2}, y_{3}$, and applying Stokes theorem to the geodesic prism with the triangles as bases, we obtain a relation involving the integral of $\omega$ over two triangles and three quadrilaterals, namely

$$
\begin{aligned}
\beta_{G}\left(y_{1}, y_{2}, y_{3}\right)= & \beta_{G}\left(x_{1}, x_{2}, x_{3}\right)+\square\left(y_{1}, y_{2}, x_{2}, x_{1}\right) \\
& +\square\left(y_{2}, y_{3}, x_{3}, x_{2}\right)+\square\left(y_{3}, y_{1}, x_{1}, x_{3}\right) .
\end{aligned}
$$

Using repeatedly the cocycle identity for $\beta_{G}$, one checks that this relation extends to all 6 -tuples of pairwise transverse points in $\overline{\mathcal{X}^{b}}$.

Denoting by $0 \in \mathcal{X}^{b}$ the origin in the ball model of $\mathcal{X}$, and setting $y_{i}:=g_{i} 0, x_{i}:=g_{i} b$ and

$$
\gamma(g, h):=\square(g b, h b, h 0, g 0),
$$

we obtain from (6.3) for all $g_{1}, g_{2}, g_{3}$ such that $g_{1} b, g_{2} b, g_{3} b$ are pairwise transverse, that

$$
c_{G}\left(g_{1}, g_{2}, g_{3}\right)=\bar{c}\left(g_{1}, g_{2}, g_{3}\right)+d \gamma\left(g_{1}, g_{2}, g_{3}\right) .
$$

Since for almost every $\left(g_{1}, g_{2}\right) \in G^{2}, g_{1} b, g_{2} b$ are transverse and

$$
\left|\gamma\left(g_{1}, g_{2}\right)\right| \leqslant 2 \pi p
$$

we deduce that $\gamma$ defines a $G$-invariant cochain in $L^{\infty}\left(G^{2}\right)$, and since (6.5) holds almost everywhere, we deduce that $c_{G}$ and $\bar{c}$ are cohomologous.

\section{Boundary maps}

We begin by recalling how to construct, from a presentation of a finitely generated group, a Poisson boundary with useful ergodicity properties. The statement was proven in $[8$, Theorem 3 and Section 2.5] and it can be proven in greater generality, namely for all compactly generated groups, using [8, Theorem 3 and Section 2.5] together with [23] (see [9, Theorem 6]). We recall its proof here in this simpler case for sake of completeness.

Proposition 7.1 [8]. - Let $\Gamma$ be a finitely generated group, $S$ a finite generating set and $(B, \nu)$ the Poisson boundary associated to the measure

$$
\mu:=\frac{1}{2|S|} \sum_{s \in S}\left(\delta_{s}+\delta_{s^{-1}}\right) .
$$

Then the diagonal action of $\Gamma$ on $B \times B$ is ergodic and the $\Gamma$-action on $B$ is amenable.

Proof. - Let $\mathbb{F}_{S}$ be the free group on the set $S, \rho: \mathbb{F}_{S} \rightarrow \Gamma$ the associated presentation of $\Gamma, \mathcal{T}_{S}$ the Cayley graph of $\mathbb{F}_{S}$ relative to $S$ and $\mathcal{T}_{s}(\infty)$ the boundary of $\mathcal{T}_{S}$. Then $\mathcal{T}_{S}(\infty)$ consists of all reduced words of infinite length and carries a natural $\mathbb{F}_{S}$-quasi-invariant measure $\bar{m}$ defined by $\bar{m}(C(x)):=\left(2 r(2 r-1)^{n-1}\right)^{-1}$, where $r=|S|, n$ is the length of $x$, and $C(x)$ consists of 
all infinite reduced words starting with $x$. It is a classical fact that $\left(\mathcal{T}_{S}(\infty), \bar{m}\right)$ is the Poisson boundary for the probability measure on $\mathbb{F}_{S}$

$$
m:=\frac{1}{2|S|} \sum_{s \in S}\left(\delta_{s}+\delta_{s^{-1}}\right) \in \mathcal{M}^{1}\left(\mathbb{F}_{S}\right) .
$$

Moreover the $\mathbb{F}_{S}$-action on $\mathcal{T}_{S}(\infty)$ is amenable, and the $\mathbb{F}_{S}$-action on $\mathcal{T}_{S}(\infty) \times \mathcal{T}_{S}(\infty)$ is ergodic. Let $N=\operatorname{ker} \rho$ and $(B, \nu)$ be the point realization of the measure algebra associated to the subalgebra $L^{\infty}\left(\mathcal{T}_{S}(\infty)\right)^{N}$ of $N$-invariant functions in $L^{\infty}\left(\mathcal{T}_{S}(\infty)\right)$. That is, $(B, \nu)$ is a standard measure space equipped with a measurable map $p: \mathcal{T}_{s}(\infty) \rightarrow B$ such that $p_{*}(\bar{m})=\nu$ and the pull-back via $p$ identifies $L^{\infty}(B, \nu)$ with $L^{\infty}\left(\mathcal{T}_{S}(\infty)\right)^{N}$. Then $\mathbb{F}_{S}$ acts on $B$, and this action factors via $\rho: \mathbb{F}_{S} \rightarrow \Gamma$. Using now that the pull-back via $\rho$ identifies $\mu=\rho_{*}(m)$-harmonic bounded functions on $\Gamma$ with $N$-invariant $m$-harmonic bounded functions on $\mathbb{F}_{S}$, we deduce that $(B, \nu)$ is a Poisson boundary for $(\Gamma, \mu)$. Since $\mathbb{F}_{S}$ acts ergodically on $\mathcal{T}_{S}(\infty) \times \mathcal{T}_{S}(\infty)$, we deduce that $\Gamma$ acts ergodically on $B \times B$. The amenability of the $\Gamma$-action on $B$ follows from a general result in [35], but can also be deduced directly by using the characterization of amenable actions given in [9]. We follow this last approach and we shall thus prove that the Banach $\Gamma$-module $L^{\infty}(B, \nu)$ is relatively injective. To this end, let $A, B$ be Banach $\Gamma$ modules, $i: A_{1} \rightarrow A_{2}$ an admissible injective $\Gamma$-morphism (see [9]) and $\alpha: A_{1} \rightarrow L^{\infty}(B, \nu)$ a $\Gamma$-morphism. Let $j: L^{\infty}(B, \nu) \rightarrow L^{\infty}\left(\mathcal{T}_{S}(\infty)\right)$ be the injection given by the pull-back via $p: \mathcal{T}_{S}(\infty) \rightarrow B$. Considering $A_{1}, A_{2}$ as Banach $\mathbb{F}_{S}$-modules via $\rho: \mathbb{F}_{S} \rightarrow \Gamma$, and $i, j \circ \alpha$ as $\mathbb{F}_{S^{-}}$ morphisms, the amenability of the $\mathbb{F}_{S}$-action on $\mathcal{T}_{S}(\infty)$ implies that $L^{\infty}\left(\mathcal{T}_{S}(\infty)\right)$ is relatively injective and hence there exists an $\mathbb{F}_{S}$-morphism $\beta: A_{2} \rightarrow L^{\infty}\left(\mathcal{T}_{S}(\infty)\right)$ extending $j \circ \alpha$. Since the $N$-action on $A_{2}$ is trivial, $\beta\left(A_{2}\right) \subset j\left(L^{\infty}(B, \nu)\right)=L^{\infty}\left(\mathcal{T}_{S}(\infty)\right)^{N} \subset L^{\infty}(\mathcal{T}(\infty))$, and hence $\left(\left.j\right|_{L^{\infty}(\mathcal{T}(\infty))^{N}}\right)^{-1} \circ \beta: A_{2} \rightarrow L^{\infty}(B, \nu)$ is a $\Gamma$-morphism extending $\alpha$.

Let now $\Gamma$ be a finitely generated group, $\pi: \Gamma \rightarrow \mathrm{SU}(V,\langle\cdot, \cdot\rangle)$ a representation, and $(B, \nu)$ a Poisson boundary of $\Gamma$ as in Proposition 7.1. Our objective is to prove:

Proposition 7.2. - Assume that $\pi(\Gamma)$ is Zariski dense. Then there exists a $\Gamma$-equivariant measurable map

$$
\varphi: B \rightarrow \mathrm{Is}_{\langle\cdot, \cdot\rangle}
$$

such that for almost all $b_{1}, b_{2} \in B, \varphi\left(b_{1}\right)$ and $\varphi\left(b_{2}\right)$ are transverse.

The proof of Proposition 7.2 is based on the following fact, whose proof we postpone.

THEOREM 7.3. - Let $\mathbf{G}$ be a connected semisimple group defined over $\mathbb{R}, \mathbf{P}$ a minimal parabolic subgroup defined over $\mathbb{R}$ and $T: \Lambda \rightarrow \mathbf{G}(\mathbb{R})$ a homomorphism of a group $\Lambda$ with Zariski dense image. Then the $\Lambda$-action on $\mathbf{G}(\mathbb{R}) / \mathbf{P}(\mathbb{R})$ is mean proximal.

We present first the proof of Proposition 7.2 assuming Theorem 7.3.

Proof of Proposition 7.2. - Let $\mathbf{G}$ be the complexification of $\mathrm{SU}(V,\langle\cdot, \cdot\rangle)$ as described in Section 3.2. In particular, we identify $\mathrm{SU}(V,\langle\cdot, \cdot\rangle)$ with $\mathbf{G}(\mathbb{R})$ and $\operatorname{Is}_{\langle\cdot, \cdot,\rangle}$ with $\operatorname{Is}_{F}(\mathbb{R})$. Pick a basepoint $b \in \operatorname{Is}_{F}(\mathbb{R})$; then $\mathbf{Q}=\operatorname{Stab}_{\mathbf{G}}(b)$ is an $\mathbb{R}$-parabolic subgroup of $\mathbf{G}$, since $\operatorname{Is}_{F}$ is a projective variety and $\mathbf{G}$ is transitive. Let $\mathbf{P}$ be a minimal parabolic subgroup of $\mathbf{G}$ defined over $\mathbb{R}$ and contained in $\mathbf{Q}$. Then we have an equivariant surjection

$$
\mathbf{G}(\mathbb{R}) / \mathbf{P}(\mathbb{R}) \rightarrow \mathbf{G}(\mathbb{R}) / \mathbf{Q}(\mathbb{R})=\mathrm{Is}_{\langle\cdot, \cdot\rangle}
$$


Since the action of $\Gamma$ on $B$ is amenable, there exists a $\Gamma$-equivariant measurable map

$$
\varphi: B \rightarrow \mathcal{M}^{1}(\mathbf{G}(\mathbb{R}) / \mathbf{P}(\mathbb{R})) .
$$

Since the $\Gamma$-action on $\mathbf{G}(\mathbb{R}) / \mathbf{P}(\mathbb{R})$ is mean proximal, $\nu$ is $\mu$-stationary, and $\operatorname{supp} \mu$ generates $\Gamma$ (see Proposition 7.1 and Theorem 7.3), [24, Corollary 2.10, p. 201] implies that for almost all $b \in B, \varphi(b)$ is a Dirac measure, thus providing a $\Gamma$-equivariant measurable map into $\mathbf{G}(\mathbb{R}) / \mathbf{P}(\mathbb{R})$, whose composition with the projection $\mathbf{G}(\mathbb{R}) / \mathbf{P}(\mathbb{R}) \rightarrow \mathbf{G}(\mathbb{R}) / \mathbf{Q}(\mathbb{R})$ gives a $\Gamma$-equivariant measurable map

$$
\varphi: B \rightarrow \mathrm{Is}_{\langle\cdot, \cdot\rangle} .
$$

We are left to show that the images under $\varphi$ of almost every two points in $B$ are transverse. To this purpose, observe that the $\Gamma$-invariant measurable map

$$
\begin{aligned}
B \times B & \rightarrow \mathbb{N} \\
\left(b_{1}, b_{2}\right) & \mapsto \operatorname{dim}\left(\varphi\left(b_{1}\right) \cap \varphi\left(b_{2}\right)\right)
\end{aligned}
$$

is essentially constant, since $\Gamma$ acts ergodically on $B \times B$. Assume that this constant is nonzero, and let $\operatorname{Ess} \operatorname{Im}(\varphi) \subset \operatorname{Is}\langle\cdot, \cdot\rangle$ be the essential image of $\varphi$. Then $\operatorname{Ess} \operatorname{Im}(\varphi)$ is closed and $\pi(\Gamma)$-invariant. For every $x \in \mathrm{Is}_{F}=\mathbf{G} / \mathbf{P}$, denoting by nt $(x)$ the set of $y \in \mathbf{G} / \mathbf{Q}$ which are non-transverse to $x$ (see Section 3), we have for almost all $b_{1} \in B$ that $\varphi\left(b_{2}\right) \in \operatorname{nt}\left(\varphi\left(b_{1}\right)\right)$ for almost every $b_{2} \in B$. Since $\operatorname{nt}(x)$ is Zariski-closed, and hence Hausdorff-closed, we have $\operatorname{Ess} \operatorname{Im}(\varphi) \subset \operatorname{nt}\left(\varphi\left(b_{1}\right)\right)$ for almost all $b_{1}$, and hence for some fixed $b_{1} \in B$. Since $\operatorname{Ess} \operatorname{Im}(\varphi)$ is $\pi(\Gamma)$-invariant, we have the inclusion

$$
\operatorname{Ess} \operatorname{Im}(\varphi) \subset \bigcap_{\gamma \in \Gamma} \pi(\gamma) \operatorname{nt}\left(\varphi\left(b_{1}\right)\right):=L
$$

Since $\operatorname{nt}\left(\varphi\left(b_{1}\right)\right)$ is a proper Zariski closed subset of $\mathbf{G} / \mathbf{P}, L$ is a proper, non-void, $\pi(\Gamma)$-invariant Zariski closed subset of $\mathbf{G} / \mathbf{Q}$ which contradicts the Zariski density of $\pi(\Gamma)$ in $\mathbf{G}$.

We now turn to the proof of Theorem 7.3. We may clearly replace $\Lambda$ by its image $T(\Lambda)$, so that now $\Lambda$ is a Zariski dense subgroup of $\mathbf{G}(\mathbb{R})$. We intend to show that the $\Lambda$-action on $\mathbf{G}(\mathbb{R}) / \mathbf{P}(\mathbb{R})$ is mean proximal by verifying the hypotheses of [24, Proposition $2.13, \mathrm{p}$. 201]. This will rely in an essential way on the following

THEOREM 7.4 [3,29]. - If $\Lambda$ is a Zariski dense subgroup of $\mathbf{G}(\mathbb{R})$, then $\Lambda$ contains an $\mathbb{R}$-regular element.

We shall need the existence, shown in [30], of a representation $\rho: \mathbf{G} \rightarrow \mathrm{GL}(W)$, defined over $\mathbb{R}$, with the following properties:

(a) An element $g \in \mathbf{G}(\mathbb{R})$ is $\mathbb{R}$-regular if and only if $\left.\rho(g)\right|_{W(\mathbb{R})}$ has a unique eigenvalue $\lambda_{g}$ of maximal modulus which occurs with multiplicity one. Let $x_{g}$ be the corresponding eigenline in the real points $\mathbb{P} W(\mathbb{R})$ of the projective space $\mathbb{P} W$;

(b) there is $x_{0} \in \mathbb{P} W(\mathbb{R})$ such that $\mathbf{P}=\operatorname{Stab}_{\mathbf{G}}\left(x_{0}\right)$ is a minimal parabolic subgroup defined over $\mathbb{R},\left\{\rho(g) x_{0}: g \in \mathbf{G}\right\}$ spans $W$, and for any $\mathbb{R}$-regular element $p \in \mathbf{P}(\mathbb{R}), x_{p}=x_{0}$.

Identifying $\mathbf{G} / \mathbf{P}$ with $\rho(\mathbf{G}) x_{0} \subset \mathbb{P} W$, and, analogously, $\mathbf{G}(\mathbb{R}) / \mathbf{P}(\mathbb{R})$ with $\rho(\mathbf{G}(\mathbb{R})) x_{0} \subset \mathbb{P} W$, we deduce from (b) and the fact that every $\mathbb{R}$-regular element $g \in \mathbf{G}(\mathbb{R})$ is conjugate to one in $\mathbf{P}(\mathbb{R})$, that $x_{g} \in \mathbf{G}(\mathbb{R}) / \mathbf{P}(\mathbb{R})$. Finally, let $W_{g} \subset W$ be the sum of all eigenspaces of $\rho(g)$ corresponding to eigenvalues of modulus less than $\lambda_{g}$. 
LEMMA 7.5. $-\Lambda$ acts strongly proximally on $\mathbf{G}(\mathbb{R}) / \mathbf{P}(\mathbb{R})$.

Proof. - (Compare with the proof of [24, Theorem 3.7, p. 205].) Let $\lambda \in \Lambda$ be an $\mathbb{R}$ regular element (see Theorem 7.4). Then $\rho(\lambda)$ attracts $\mathbf{G}(\mathbb{R}) / \mathbf{P}(\mathbb{R}) \backslash \mathbb{P} W_{\lambda}(\mathbb{R})$ towards $x_{\lambda}$. For $x \in \mathbf{G}(\mathbb{R}) / \mathbf{P}(\mathbb{R})$ define

$$
\mathbf{G}_{x}:=\left\{h \in \mathbf{G}: \rho(h) x \in \mathbb{P} W_{\lambda} \cap \mathbf{G} / \mathbf{P}\right\} .
$$

Since $\mathbb{P} W_{\lambda} \cap \mathbf{G} / \mathbf{P}$ is a proper Zariski closed subset of $\mathbf{G} / \mathbf{P}$, the set $\mathbf{G}_{x}$ is a proper Zariski closed subset of $\mathbf{G}$, and hence, since $\bar{\Lambda}^{Z}=\mathbf{G}$, for $y, z \in \mathbf{G}(\mathbb{R}) / \mathbf{P}(\mathbb{R})$ fixed, there exists $\mu \in \Lambda$ with $\rho(\mu) y \notin \mathbb{P} W_{\lambda}$ and $\rho(\mu) z \notin \mathbb{P} W_{\lambda}$. Thus

$$
\lim _{n \rightarrow \infty} \rho\left(\lambda^{n} \mu\right) y=x_{\lambda}
$$

and

$$
\lim _{n \rightarrow \infty} \rho\left(\lambda^{n} \mu\right) z=x_{\lambda}
$$

which proves that $\Lambda$ acts proximally on $\mathbf{G}(\mathbb{R}) / \mathbf{P}(\mathbb{R})$. To deduce that $\Lambda$ acts strongly proximally, we proceed to show that every point in $\mathbf{G}(\mathbb{R}) / \mathbf{P}(\mathbb{R})$ has a contractible neighborhood; the lemma will then follow from [24, Proposition 1.6(a), p. 196]. Since $\rho(\mu) W_{\lambda}=W_{\mu \lambda \mu^{-1}}$, the subvariety of $\mathbf{G} / \mathbf{P}$

$$
\left(\bigcap_{\mu \in \Lambda} \rho(\mu) \mathbb{P} W_{\lambda}\right) \cap \mathbf{G} / \mathbf{P}
$$

is $\Lambda$-invariant and hence $\mathbf{G}$-invariant. It is also properly contained in $\mathbf{G} / \mathbf{P}$ and hence void. This implies that

$$
\bigcup_{\mu \in \Lambda} \rho(\mu) \mathbb{P} W_{\lambda}^{c} \supset \mathbf{G} / \mathbf{P}
$$

and hence that every point in $\mathbf{G}(\mathbb{R}) / \mathbf{P}(\mathbb{R})$ is contained in the contractible open set

$$
\mathbf{G}(\mathbb{R}) / \mathbf{P}(\mathbb{R}) \backslash \mathbb{P} W_{\mu \lambda \mu^{-1}},
$$

for some $\mu$.

Let $d$ be a distance on $\mathbb{P} W(\mathbb{R})$ and define (see [24, p. 203]) $\Psi_{\varepsilon}$ to be the family of subsets of $\mathbb{P} W(\mathbb{R})$ of the form

$$
W^{\prime}(\varepsilon):=\left\{x \in \mathbb{P} W(\mathbb{R}): d\left(x, \mathbb{P} W^{\prime}(\mathbb{R})\right)>\varepsilon\right\},
$$

where $W^{\prime} \in \mathrm{Gr}_{m-1}(W)$ is defined over $\mathbb{R}$ for $m=\operatorname{dim} W$. In view of [24, Lemma 3.2, p. 203], in order to verify [24, Proposition 2.13(b)], we need only to show the following

LEMMA 7.6. - There exists $\varepsilon>0$ such that for every $W^{\prime}(\varepsilon) \in \Psi_{\varepsilon}$,

$$
\rho(\Lambda)\left(W^{\prime}(\varepsilon) \cap \mathbf{G}(\mathbb{R}) / \mathbf{P}(\mathbb{R})\right)=\mathbf{G}(\mathbb{R}) / \mathbf{P}(\mathbb{R}) .
$$

Proof. - For every $W^{\prime}(\mathbb{R}) \in \mathrm{Gr}_{m-1}(W(\mathbb{R})), x \in \mathbf{G}(\mathbb{R}) / \mathbf{P}(\mathbb{R})$, define

$$
f\left(x, W^{\prime}(\mathbb{R})\right)=\sup _{\lambda \in \Lambda} d\left(\rho(\lambda) x, \mathbb{P} W^{\prime}(\mathbb{R}) \cap \mathbf{G}(\mathbb{R}) / \mathbf{P}(\mathbb{R})\right) .
$$


If $f\left(x, W^{\prime}(\mathbb{R})\right)$ were to vanish, then we would have that $\rho(\Lambda) x \subset \mathbb{P} W^{\prime} \cap \mathbf{G} / \mathbf{P}$, and hence $\rho(\mathbf{G}) x \subset \mathbb{P} W^{\prime} \cap \mathbf{G} / \mathbf{P}$, which would imply that $\mathbb{P} W^{\prime} \supset \mathbf{G} / \mathbf{P}$ and hence

$$
W^{\prime} \supset\left\{\rho(g) x_{0}: g \in \mathbf{G}\right\} .
$$

Since the latter spans $W$ we would obtain a contradiction. The lower semicontinuous function $f$ is hence positive on the compact space $\mathbf{G}(\mathbb{R}) / \mathbf{P}(\mathbb{R}) \times \mathrm{Gr}_{m-1}(W(\mathbb{R}))$ and thus there exists $\varepsilon>0$ such that $f\left(x, W^{\prime}(\mathbb{R})\right)>\varepsilon$ for all $x \in \mathbf{G}(\mathbb{R}) / \mathbf{P}(\mathbb{R})$ and $W^{\prime}(\mathbb{R}) \in \mathrm{Gr}_{m-1}(W(\mathbb{R}))$. This implies the lemma.

\section{A formula for the bounded Kähler class of a representation}

Let $\Gamma$ be a finitely generated group and $(B, \nu)$ the Poisson boundary given in Proposition 7.1. Then the complex

$$
\mathbb{R} \longrightarrow L^{\infty}(B) \stackrel{d}{\longrightarrow} L_{\text {alt }}^{\infty}\left(B^{2}\right) \stackrel{d}{\longrightarrow} \cdots,
$$

is a relatively injective resolution of $\mathbb{R}$ and hence the bounded cohomology $\mathrm{H}_{\mathrm{b}}^{\bullet}(\Gamma, \mathbb{R})$ is canonically isomorphic to the cohomology of the non-augmented subcomplex of invariants $\left(L_{\text {alt }}^{\infty}\left(B^{\bullet}\right)^{\Gamma}, d_{\bullet}\right)$. Together with the ergodicity of the $\Gamma$-action on $B \times B$, this yields an isomorphism of Banach spaces

$$
\mathrm{H}_{\mathrm{b}}^{2}(\Gamma, \mathbb{R}) \stackrel{\simeq}{\rightarrow} \mathcal{Z} L_{\mathrm{alt}}^{\infty}\left(B^{3}\right)^{\Gamma}
$$

where the right-hand side is the space of $\Gamma$-invariant, alternating, essentially bounded cocycles on $B^{3}$. Let now $\pi: \Gamma \rightarrow G=\mathrm{SU}(V,\langle\cdot, \cdot\rangle)$ be a representation with Zariski dense image,

$$
\varphi: B \rightarrow \mathrm{Is}_{\langle\cdot, \cdot\rangle}
$$

the $\Gamma$-equivariant map given by Proposition 7.2 and $\psi$ its composition with $E: \mathrm{Is}_{\langle\cdot, \cdot\rangle} \rightarrow \check{S}$. Then it follows from Proposition 7.2 that for almost every $\left(b_{1}, b_{2}, b_{3}\right) \in B^{3}, \psi\left(b_{1}\right), \psi\left(b_{2}\right), \psi\left(b_{3}\right)$ are pairwise transverse and hence

$$
\psi_{3}^{*} \beta_{G}\left(b_{1}, b_{2}, b_{3}\right):=\beta_{G}\left(\psi\left(b_{1}\right), \psi\left(b_{2}\right), \psi\left(b_{3}\right)\right)
$$

is a well defined element in $\mathcal{Z} L_{\text {alt }}^{\infty}\left(B^{3}\right)^{\Gamma}$.

THEOREM 8.1. - Under the isomorphism

$$
\mathrm{H}_{\mathrm{b}}^{2}(\Gamma, \mathbb{R}) \stackrel{\simeq}{\rightarrow} \mathcal{Z} L_{\mathrm{alt}}^{\infty}\left(B^{3}\right)^{\Gamma},
$$

$\pi^{*}\left(\kappa_{G}^{\mathrm{b}}\right)$ corresponds to $\psi_{3}^{*} \beta_{G}$.

Proof. - We shall use the resolution defined in Section 6 on the Shilov boundary $\check{S}$. Let

$$
\psi^{n}: B^{n} \rightarrow \check{S}^{n}
$$

be the $n$th Cartesian product of the map $\psi$. Since $\psi\left(b_{1}\right), \psi\left(b_{2}\right)$ are transverse for almost every $\left(b_{1}, b_{2}\right) \in B^{2}$, we deduce that $\psi^{n}\left(b_{1}, \ldots, b_{n}\right) \in \check{S}^{(n)}$ for almost every $\left(b_{1}, \ldots, b_{n}\right) \in B^{n}$. Thus, for $f \in \mathcal{B}_{\text {alt }}^{\infty}\left(\check{S}^{(n)}\right)$, we define

$$
\psi_{n}^{*}(f)\left(b_{1}, \ldots, b_{n}\right):=f \psi^{n}\left(b_{1}, \ldots, b_{n}\right) .
$$


and obtain in this way a morphism of complexes

$$
\psi_{\bullet}^{*}: \mathcal{B}_{\mathrm{alt}}^{\infty}\left(\check{S}^{(\bullet)}\right) \rightarrow L_{\mathrm{alt}}^{\infty}\left(B^{\bullet}\right)
$$

extending the identity $\mathbb{R} \rightarrow \mathbb{R}$. Using that the complex of continuous bounded functions $C_{\mathrm{b}}\left(G^{\bullet}\right)$ on $G^{n}, n \geqslant 1$, gives a strong resolution by relatively injective modules, we obtain a morphism of complexes

$$
\alpha_{\bullet}: \mathcal{B}_{\mathrm{alt}}^{\infty}\left(\check{S}^{(\bullet)}\right) \rightarrow C_{\mathrm{b}}\left(G^{\bullet}\right) .
$$

Finally, let $\pi_{n}: C_{\mathrm{b}}\left(G^{n}\right) \rightarrow \ell^{\infty}\left(\Gamma^{n}\right)$ be the morphism of complexes given by the precomposition with $\pi: \Gamma \rightarrow G$. It follows then from [6, Proposition 1.2] that the diagram in cohomology

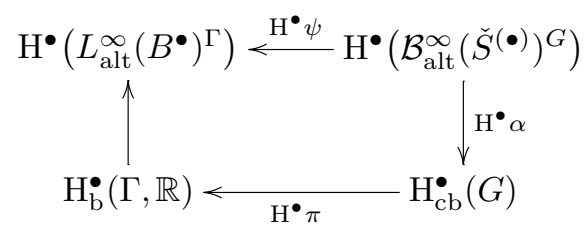

commutes. Since by Lemma $6.2, \mathrm{H}^{2} \alpha\left(\left[\beta_{G}\right]\right)=\kappa_{G}^{\mathrm{b}}$ and $\mathrm{H}^{2} \psi\left(\left[\beta_{G}\right]\right)$ is represented by $\psi_{3}^{*} \beta_{G}$, the theorem is proven.

\section{The proofs}

Proof of Theorems 1.1 and 1.3. - Let $\pi_{i}: \Gamma \rightarrow \operatorname{PSU}\left(V,\langle\cdot, \cdot\rangle_{i}\right)$ be homomorphisms with Zariski dense image, and $\kappa_{i}^{\mathrm{b}}$ the bounded Kähler class of $\operatorname{PSU}\left(V,\langle\cdot, \cdot\rangle_{i}\right)$. Since

$$
G_{i}:=\mathrm{SU}\left(V,\langle\cdot, \cdot\rangle_{i}\right) \stackrel{a_{i}}{\rightarrow} \operatorname{PSU}\left(V,\langle\cdot, \cdot\rangle_{i}\right)
$$

is a finite central extension of $\operatorname{PSU}\left(V,\langle\cdot, \cdot\rangle_{i}\right)$, there exist a finite central extension of $\Gamma$

$$
\tilde{\Gamma} \stackrel{a}{\rightarrow} \Gamma
$$

and homomorphisms

$$
\tilde{\pi}_{i}: \tilde{\Gamma} \rightarrow \mathrm{SU}\left(V,\langle\cdot, \cdot\rangle_{i}\right)
$$

with Zariski dense image such that $a_{i} \tilde{\pi}_{i}=\pi_{i} a$. Assume now that there are $m_{i} \in \mathbb{Z}$ not all zero with

$$
\sum_{i=1}^{r} m_{i} \pi_{i}^{*}\left(\kappa_{i}^{\mathrm{b}}\right)=0 .
$$

Denoting with $\tilde{\kappa}_{i}^{\mathrm{b}}$ the bounded Kähler class of $\mathrm{SU}\left(V,\langle\cdot, \cdot\rangle_{i}\right)$, and observing that $a_{i}^{*}\left(\kappa_{i}^{\mathrm{b}}\right)=\tilde{\kappa}_{i}^{\mathrm{b}}$, we obtain

$$
\sum_{i=1}^{r} m_{i} \tilde{\pi}_{i}^{*}\left(\tilde{\kappa}_{i}^{\mathrm{b}}\right)=0 .
$$

Let $B$ be the Poisson boundary associated to a presentation of $\tilde{\Gamma}$ (see Proposition 7.1) and

$$
\varphi_{i}: B \rightarrow \operatorname{Is}_{F_{i}}(\mathbb{R}) \subset \operatorname{Is}_{F_{i}}
$$


the boundary map given by Proposition 7.2. Taking into account Theorem 8.1, (5.6) and (9.1), we obtain that for almost all $\left(b_{1}, b_{2}, b_{3}\right) \in B^{3}$ and all $1 \leqslant i \leqslant r,\left(\varphi_{i}\left(b_{1}\right), \varphi_{i}\left(b_{2}\right), \varphi_{i}\left(b_{3}\right)\right) \in \operatorname{Is}_{F_{i}}^{(3)}$ and

$$
\prod_{i=1}^{r}\left\langle\varphi_{i}\left(b_{1}\right), \varphi_{i}\left(b_{2}\right), \varphi_{i}\left(b_{3}\right)\right\rangle_{\mathbb{C}}^{m_{i}}=[1] \quad \text { in } \mathbb{C}^{\times} \backslash A^{\times} .
$$

Consider now the measurable map

$$
\begin{aligned}
\varphi: B & \rightarrow \prod_{i=1}^{r} \operatorname{Is}_{F_{i}}(\mathbb{R}) \\
b & \mapsto\left(\varphi_{i}(b)\right)_{i},
\end{aligned}
$$

$\tilde{\Gamma}$-equivariant with respect to the representation

$$
\begin{aligned}
\tilde{\pi}: \tilde{\Gamma} & \rightarrow \prod_{i=1}^{r} \mathbf{G}_{i} \\
\gamma & \mapsto\left(\tilde{\pi}_{i}(\gamma)\right)_{i},
\end{aligned}
$$

define $\Lambda=\tilde{\pi}(\tilde{\Gamma})$ and let $\mathbf{H}=\bar{\Lambda}^{Z}$ be the Zariski closure of $\Lambda$ in $\prod_{i=1}^{r} \mathbf{G}_{i}$. Observe that, because $\varphi$ is $\tilde{\Gamma}$-equivariant, its essential image $\operatorname{Ess} \operatorname{Im}(\varphi)$ is $\Lambda$-invariant. Fix now $\left(b_{1}, b_{2}\right) \in B^{2}$ such that (9.2) holds for almost every $b_{3} \in B$. In the notation of Lemma 4.4 and the paragraph preceding it, set

$$
\begin{aligned}
P_{i} & :=P_{\varphi_{i}\left(b_{1}\right), \varphi_{i}\left(b_{2}\right)}, \\
\mathcal{O}_{i} & :=\mathcal{O}_{\varphi_{i}\left(b_{1}\right), \varphi_{i}\left(b_{2}\right)}, \quad \text { and } \quad \mathcal{O}=\prod_{i=1}^{r} \mathcal{O}_{i} .
\end{aligned}
$$

Then it follows from (9.2) that

$$
\operatorname{Ess} \operatorname{Im}(\varphi) \cap \mathcal{O} \subset\left\{\left(x_{1}, \ldots, x_{r}\right) \in \prod_{i=1}^{r} \mathcal{O}_{i}: \prod_{i=1}^{r} P_{i}\left(x_{i}\right)^{m_{i}}=1\right\} .
$$

In view of Lemma 4.4, the latter is a Zariski closed proper subset of $\prod_{i=1}^{r} \mathcal{O}_{i}$, which implies that $\operatorname{Ess} \operatorname{Im}(\varphi)$ is contained in a Zariski closed proper subset of $\prod_{i=1}^{r} \operatorname{Is}_{F_{i}}$, and hence that $\overline{\operatorname{Ess} \operatorname{Im}(\varphi)} Z$ is a $\Lambda$-invariant Zariski closed proper subset. This subset is thus $\mathbf{H}$-invariant, which implies that $\mathbf{H}$ is a proper $\mathbb{R}$-algebraic subgroup of $\prod_{i=1}^{r} \mathbf{G}_{i}$. If we denote by $Z\left(\mathbf{G}_{i}\right)$ the center of $\mathbf{G}_{i}$, let now

$$
\mathbf{L}_{i}:=\mathbf{G}_{i} / Z\left(\mathbf{G}_{i}\right) \quad \text { and } \quad \mathbf{L}:=\prod_{i=1}^{r} \mathbf{L}_{i},
$$

and let $\mathbf{D}$ be the image of $\mathbf{H}$ in $\mathbf{L}$. Then, under the identification of $\operatorname{PSU}\left(V,\langle\cdot, \cdot\rangle_{i}\right)$ with $\mathbf{L}_{i}(\mathbb{R})$, we deduce from $\Lambda<\mathbf{H}$ that

$$
\left\{\left(\pi_{1}(\gamma), \ldots, \pi_{r}(\gamma)\right): \gamma \in \Gamma\right\}<\mathbf{D}(\mathbb{R}) .
$$

Observe first that since $\mathbf{D} \neq \mathbf{L}$, the case $r=1$ cannot occur, which implies the first assertion of Theorem 1.1. Thus $r \geqslant 2$. Since the $\mathbf{L}_{i}$ 's are simple (as abstract groups), non-abelian, 
$\operatorname{pr}_{i}(\mathbf{D})=\mathbf{L}_{i}$ and $\mathbf{D} \lesseqgtr \mathbf{L}$, the subgroup $\mathbf{D}$ determines a partition $I_{1} \cup \cdots \cup I_{\ell}=\{1, \ldots, r\}$ with $\ell<r$, and, for every $1 \leqslant k \leqslant \ell, i, j \in I_{k}$, an isomorphism

$$
\pi_{i j}: \mathbf{L}_{i} \rightarrow \mathbf{L}_{j}
$$

defined over $\mathbb{R}$ such that

$$
\pi_{j}(\gamma)=\pi_{i j} \pi_{i}(\gamma), \quad \gamma \in \Gamma
$$

Since $\ell<r$, we have that $\left|I_{k}\right| \geqslant 2$ for some $k$ and hence there is $i \neq j$ such that $\left(\pi_{i}, \mathcal{X}_{i}\right)$ and $\left(\pi_{j}, \mathcal{X}_{j}\right)$ are equivalent, which proves Theorem 1.3.

Let $r=2, m_{1}=-m_{2}=1,\langle\cdot, \cdot\rangle_{1}=\langle\cdot, \cdot\rangle_{2}$ and $T_{12}: \mathcal{X}_{1} \rightarrow \mathcal{X}_{2}$ the isometry induced by $\pi_{12}$. Let $\varepsilon=1$ if $T_{12}$ preserves the complex structure and $\varepsilon=-1$ otherwise. Then we get

$$
\pi_{2}(\gamma)=\pi_{12} \pi_{1}(\gamma), \quad \gamma \in \Gamma,
$$

and hence $(1-\varepsilon) \pi_{1}^{*}\left(\kappa_{1}^{\mathrm{b}}\right)=0$ which, in view of Theorem 1.1(i), implies that $\varepsilon=1$ and that $T_{12}$ is holomorphic. This shows part (ii) of Theorem 1.1.

Proposition 9.1.- Let $c: I \rightarrow \mathcal{R}_{\mathrm{Zd}}(\Gamma, G)$ be any continuous injective path from an open interval $I \subset \mathbb{R}$ and let $K: \mathcal{R}_{\mathrm{Zd}}(\Gamma, G) \rightarrow \mathrm{H}_{\mathrm{b}}^{2}(\Gamma, \mathbb{R})$ be the map defined in (1.2) by $K(\pi)=\pi^{*}\left(\kappa_{G}^{\mathrm{b}}\right)$. Then $\{K(c(t)): t \in I\} \subset \mathrm{H}_{\mathrm{b}}^{2}(\Gamma, \mathbb{R})$ contains an uncountable subset which is independent over $\mathbb{R}$.

Proof of Proposition 9.1 and Corollary 1.4. - Let $\left(\ell^{\infty}\left(\Gamma^{\bullet}\right), d_{\bullet}\right)$ denote the standard (bounded) non-homogeneous complex, and $\left(\ell^{1}\left(\Gamma^{\bullet}\right), \partial_{\bullet}\right)$ the non-homogeneous complex of $\ell^{1}$-chains. In particular,

$$
\ell^{\infty}(\Gamma) \stackrel{d_{1}}{\longrightarrow} \ell^{\infty}\left(\Gamma^{2}\right)
$$

is the adjoint of

$$
\ell^{1}\left(\Gamma^{2}\right) \stackrel{\partial_{2}}{\longrightarrow} \ell^{1}(\Gamma)
$$

and, since $\mathrm{H}_{\mathrm{b}}^{2}(\Gamma, \mathbb{R})$ is a Banach space, $\operatorname{Im} d_{1} \subset \ell^{\infty}\left(\Gamma^{2}\right)$ is norm closed. Since $d_{1}=\partial_{2}^{*}$, $\operatorname{Im} d_{1}$ is thus weak-* closed and hence the weak-* topology on $\ell^{\infty}\left(\Gamma^{2}\right)$ induces a locally convex Hausdorff topology on $\mathrm{H}_{\mathrm{b}}^{2}(\Gamma, \mathbb{R})$ for which the map $K: \mathcal{R}_{\mathrm{Zd}}(\Gamma, G) \rightarrow \mathrm{H}_{\mathrm{b}}^{2}(\Gamma, \mathbb{R})$ is easily seen to be continuous. In particular, any subspace of $\mathrm{H}_{\mathrm{b}}^{2}(\Gamma, \mathbb{R})$ of finite dimension $n$ is isomorphic to $\mathbb{R}^{n}$ as a locally convex topological vector space, and hence is closed.

Let $W$ be the vector subspace generated by $\{K(c(t)): t \in I\}$ and assume that it has countable dimension. Let $W=\bigcup_{n \geqslant 1} W_{n}$, where $\left\{W_{n}\right\}$ is a sequence of increasing finite dimensional subspaces. Then $I=\bigcup_{n \geqslant 1}(K c)^{-1}\left(W_{n}\right)$ and, since $(K c)^{-1}\left(W_{n}\right)$ is closed, there is $n_{0} \geqslant 1$ such that $(K c)^{-1}\left(W_{n_{0}}\right)$ has non-void interior. Let $d=\operatorname{dim}\left(W_{n_{0}}\right)$, so that we identify $W_{n_{0}}$ with $\mathbb{R}^{d}$. Choose an open non-void interval $J \subset(K c)^{-1}\left(W_{n_{0}}\right) \subset \mathbb{R}$.

Let $\alpha \in \operatorname{Aut}(G)$ be the exterior automorphism of order 2 which reverses the complex structure on the associated symmetric space. Then $\alpha$ acts freely and properly on $\mathcal{R}_{\mathrm{Zd}}(\Gamma, G)$ thus, by shrinking $J$, we may assume that $\alpha(c(J)) \cap c(J)=\emptyset$. For any $m \geqslant 1$, let $J^{(m)}$ be the set of $m$-tuples of distinct points on $J$, and consider the map

$$
\begin{aligned}
T_{m}: J^{(m)} & \rightarrow \mathbb{R}^{d}\left(\simeq W_{n_{0}}\right) \\
\left(t_{1}, \ldots, t_{m}\right) & \mapsto \sum_{i=1}^{m} i K\left(c\left(t_{i}\right)\right) .
\end{aligned}
$$


We claim that $T_{m}$ is injective. Indeed, assume that $T_{m}(t)=T_{m}(s)$, where $t=\left(t_{1}, \ldots, t_{m}\right)$ and $s=\left(s_{1}, \ldots, s_{m}\right)$. Let $\sigma \in S_{m}$ be a permutation such that $t_{1}, \ldots, t_{s}, t_{s+1} \ldots, t_{m}, s_{\sigma(s+1)}, \ldots$, $s_{\sigma(m)}$ are pairwise distinct and $t_{i}=s_{\sigma(i)}$ for $1 \leqslant i \leqslant s$. Then $T_{m}(t)=T_{m}(s)$ implies that

$$
\sum_{i=1}^{s}(i-\sigma(i)) K\left(c\left(t_{i}\right)\right)+\sum_{i=s+1}^{m} i K\left(c\left(t_{i}\right)\right)-\sum_{i=s+1}^{m} \sigma(i) K\left(c\left(s_{\sigma(i)}\right)\right)=0,
$$

which, in view of Theorem 1.3, forces $s=m$ and $i=\sigma(i)$, that is $t=s$.

Thus, the fact that $T_{m}: J^{(m)} \rightarrow \mathbb{R}^{d}$ is a continuous injective map from the $m$-dimensional manifold $J^{(m)}$ into $\mathbb{R}^{d}$, forces $m \leqslant d$, which is a contradiction. Thus $W$ has uncountable dimension, which proves Proposition 9.1.

For the proof of Corollary 1.4, observe that the set $C$ of regular points of $\mathcal{R}_{\mathrm{Zd}}(\Gamma, G)$ is a manifold. Then, if $\mathrm{H}_{\mathrm{b}}^{2}(\Gamma, G)$ is finite dimensional, Proposition 9.1 implies that there are no continuous injective paths into $C$, and hence each connected component of $C$ is reduced to a point thus implying that $\mathcal{R}_{\mathrm{Zd}}(\Gamma, G)$ is finite.

Proof of Corollary 1.6. - Observe that if $\omega: \Gamma \rightarrow G$ is of type $(p, q)$, then, by Lemma 4.2, $\kappa_{G} / 4 \pi \operatorname{gcd}(p, q)$ is in the image of $\mathrm{H}^{2}(G, \mathbb{Z})$ under the map $\mathrm{H}^{2}(G, \mathbb{Z}) \rightarrow \mathrm{H}^{2}(G, \mathbb{R})$, and hence $\omega^{*}\left(\kappa_{G}\right) / 4 \pi \operatorname{gcd}(p, q)$ is in the image of $\mathrm{H}^{2}(\Gamma, \mathbb{Z}) \rightarrow \mathrm{H}^{2}(\Gamma, \mathbb{R})$. Letting now $\omega_{i}: \Gamma \rightarrow G_{i}$, $1 \leqslant i \leqslant n$, be inequivalent, Zariski dense representations, with $\omega_{i}: \Gamma \rightarrow G_{i}$ of type $\left(p_{i}, q_{i}\right)$, and setting

$$
c_{i}:=\frac{\omega_{i}^{*}\left(\kappa_{G_{i}}\right)}{4 \pi \operatorname{gcd}\left(p_{i}, q_{i}\right)},
$$

we deduce from Theorem 1.3 and the hypothesis that $\mathrm{H}_{\mathrm{b}}^{2}(\Gamma, \mathbb{R})$ injects into $\mathrm{H}^{2}(\Gamma, \mathbb{R})$, that the family

$$
\left\{4 \pi \operatorname{gcd}\left(p_{i}, q_{i}\right) c_{i}: 1 \leqslant i \leqslant n\right\} \subset \mathrm{H}^{2}(\Gamma, \mathbb{R})
$$

is linearly independent over $\mathbb{Z}$ and hence that the family

$$
\left\{c_{i}: 1 \leqslant i \leqslant n\right\} \subset \mathrm{H}^{2}(\Gamma, \mathbb{R})
$$

is linearly independent over $\mathbb{Z}$ as well. Since we are dealing with ordinary cohomology and all the $c_{i}$ 's are integral classes, we deduce that $\left\{c_{i}: 1 \leqslant i \leqslant n\right\}$ is linearly independent over $\mathbb{R}$.

\section{Acknowledgements}

Most of the final work for this paper was done while the authors were participating in the Research in Pairs program at the Mathematisches Forschungsinstitut in Oberwolfach. We thank the MFO for their hospitality and the "Land Baden-Würtemberg" for their support of the Institute.

Our thanks also go to Indira Chatterji and to Nicolas Monod for stimulating discussions.

\section{REFERENCES}

[1] A'Campo N., Burger M., Réseaux arithmétiques et commensurateurs d'après G. A. Margulis, Invent. Math. 116 (1994) 1-25.

[2] Benoist Y., Actions propres sur les espaces homogènes réductifs, Ann. of Math. (2) 144 (1996) 315347. 
[3] Benoist Y., Labourie F., Sur les difféomorphismes d'Anosov affines à feuilletages stable et instable différentiables, Invent. Math. 111 (1993) 285-308.

[4] Bradlow S.B., Garcia-Prada O., Gothen P.B., Surface group representations in PU $(p, q)$ and Higgs bundles, http://arxiv.org/abs/math.AG/0211431, 2002.

[5] BROOKS R., Some remarks on bounded cohomology, in: Riemann Surfaces and Related Topics: Proceedings of the 1978 Stony Brook Conference (State Univ. New York, Stony Brook, NY, 1978) (Princeton, NJ), in: Ann. of Math. Stud., vol. 97, Princeton Univ. Press, 1981, pp. 53-63.

[6] Burger M., Iozzi A., Boundary maps in bounded cohomology, Geom. Funct. Anal. 12 (2002) 281292.

[7] Burger M., IOZZI A., Wienhard A., Surface group representations with maximal Toledo invariant, C. R. Acad. Sci. Paris Sér. I 336 (2003) 387-390.

[8] Burger M., Monod N., Continuous bounded cohomology and applications, unpublished.

[9] Burger M., Monod N., Continuous bounded cohomology and applications to rigidity theory, Geom. Funct. Anal. 12 (2002) 219-280.

[10] ClerC J.L., ØRsted B., The Gromov norm of the Käehler class and the Maslov index, preprint, June 2002.

[11] Domic A., Toledo D., The Gromov norm of the Käehler class of symmetric domains, Math. Ann. 276 (3) (1987) 425-432.

[12] Dupont J.L., Bounds for Characteristic Numbers of Flat Bundles, in: Algebraic topology, Aarhus 1978, in: Lecture Notes in Mathematics, vol. 763, Springer-Verlag, 1979.

[13] Dupont J.L., Guichardet A., À propos de l'article: "Sur la cohomologie réelle des groupes de Lie simples réels", Ann. Sci. École Norm. Sup. (4) 11 (2) (1978) 277-292 par A. Guichardet et D. Wigner, Ann. Sci. École Norm. Sup. (4) 11 (2) (1978) 293-295.

[14] GHys É., Groupes d'homéomorphismes du cercle et cohomologie bornée, in: The Lefschetz Centennial Conference, Part III (Mexico City 1984), in: Contemp. Math., vol. 58, American Mathematical Society, RI, 1987, pp. 81-106.

[15] GHYs É., Le cercle à l'infini des surfaces à courbure négative, in: Proceedings of the International Congress of Mathematicians, vols. I, II (Kyoto, 1990) (Tokyo), Math. Soc. Japan, 1991, pp. 501-509.

[16] Goldman W.M., Complex Hyperbolic Geometry, in: Oxford Mathematical Monographs, The Clarendon Press Oxford University Press, New York, 1999, Oxford Science Publications.

[17] Goldman W.M., Topological components of spaces of representations, Invent. Math. 93 (3) (1988) 557-607.

[18] GRIGORCHUK R.I., Some results on bounded cohomology, in: Combinatorial and Geometric Group Theory (Edinburgh, 1993), in: London Math. Soc. Lecture Note Ser., vol. 204, Cambridge Univ. Press, Cambridge, 1995, pp. 111-163.

[19] Guichardet A., Wigner D., Sur la cohomologie réelle des groupes de Lie simples réels, Ann. Sci. École Norm. Sup. (4) 11 (2) (1978) 277-292.

[20] Gusevskit N., PARKER J.R., Representations of free Fuchsian groups in complex hyperbolic space, Topology 39 (2000) 33-60.

[21] Hernàndez Lamoneda L., Maximal representations of surface groups in bounded symmetric domains, Trans. Amer. Math. Soc. 324 (1991) 405-420.

[22] IozZI A., Bounded cohomology, boundary maps, and representations into Homeo+ $\left(S^{1}\right)$ and $\mathrm{SU}(1, n)$, in: Rigidity in Dynamics and Geometry, Cambridge, UK, 2000, Springer-Verlag, 2002, pp. 237-260.

[23] Kaimanovich V.A., The Poisson boundary of an amenable extension, Monatsh. Math. 136 (1) (2002) 9-15.

[24] Margulis G.A., Discrete Subgroups of Semisimple Lie Groups, Springer-Verlag, New York, 1991.

[25] Matsumoto S., Some remarks on foliated $S^{1}$ bundles, Invent. Math. 90 (1987) 343-358.

[26] Mitsumatsu Y., Bounded cohomology and $l^{1}$-homology of surfaces, Topology 23 (4) (1984) 465471.

[27] Monod N., Continuous Bounded Cohomology of Locally Compact Groups, in: Lecture Notes in Math., vol. 1758, Springer-Verlag, 2001.

[28] Monod N., Shalom Y., Rigidity of orbit equivalence and bounded cohomology, Ann. Math., in press. 
[29] Prasad G., $\mathbb{R}$-regular elements in Zariski-dense subgroups, Quart. J. Math. Oxford Ser. (2) 45 (180) (1994) 541-545.

[30] Prasad G., Raghunathan M.S., Cartan subgroups and lattices in semi-simple groups, Ann. of Math. 96 (1972) 296-317.

[31] Procesi C., Schwarz G., Inequalities defining orbit spaces, Invent. Math. 81 (3) (1985) 539-554.

[32] Toledo D., Harmonic maps from surfaces to certain Kähler manifolds, Math. Scand. 45 (1979) 1326.

[33] Toledo D., Representations of surface groups in complex hyperbolic space, J. Differential Geom. 29 (1) (1989) 125-133.

[34] XIA E.Z., The moduli of flat $U(p, 1)$ structures on Riemann surfaces, preprint, 2001.

[35] Zimmer R.J., Amenable ergodic group actions and an application to Poisson boundaries of random walks, J. Funct. Anal. 27 (1978) 350-372.

Marc BURGER

FIM, ETH Zentrum,

Rämistrasse 101,

CH-8092 Zürich, Switzerland

E-mail: burger@math.ethz.ch

\section{Alessandra IoZZI \\ ETH Zentrum,}

Departement Mathematik,

Rämistrasse 101,

CH-8092 Zürich, Switzerland

E-mail: iozzi@math.ethz.ch 